\title{
Development of Topographic Order in the Mammalian Retinocollicular Projection
}

\author{
David K. Simon and Dennis D. M. O'Leary \\ Molecular Neurobiology Laboratory, The Salk Institute, La Jolla, California 92037
}

We have used the anterograde axon tracer $1,1^{\prime}$-dioctodecyl$3,3,3^{\prime}, 3^{\prime}$-tetramethylindocarbocyanine perchlorate (Dil) to characterize the development of topographic order in the rat retinocollicular projection. Retinal axons were labeled by Dil injections covering $\mathbf{0 . 1 5 - 2} \%$ of peripheral temporal, nasal, superior, or inferior retina, or more central retina, in rats ranging in age from embryonic day 20 to postnatal day (P) 19. At $P 11-P 12$ and later, such injections label retinal axons that form overlapping arbors restricted to a topographically correct terminal zone covering about $1 \%$ of the superior colliculus (SC) area. At perinatal ages, though, axons labeled from each retinal site are distributed in the SC over much of its medial-lateral axis and extend caudally well beyond the rostral-caudal location of their correct terminal zone; some continue caudally into the inferior colliculus. Axons typically form side branches and often arborize at topographically incorrect positions throughout the SC; however, they appear to branch preferentially in a region that includes, but is much larger than, their correct terminal zone. The mature, retinotopically ordered projection emerges during an early postnatal remodeling period through the rapid remodeling of the early, diffuse projection. This process involves the largescale removal of axons, axon segments, branches, and arbors from topographically inappropriate positions concurrently with a dramatic increase in branching and arborization at topographically correct locations. Quantitative measurements show that elimination of aberrant branches without loss of the primary axons contributes substantially to the development of order. By P6, fewer mistargeted axons persist, but those that do persist tend to branch or arborize more extensively in topographically inappropriate regions. By P8, the labeling patterns begin to approximate those seen at maturity. Further refinement leads to an adultlike topographic ordering of axonal arborizations by P11-P12. At maturity, some axons take very indirect routes to reach their correct terminal zone. However, such trajectory changes typically correct only small positional inaccuracies, indicating that axons and axon segments that make larger targeting errors do not survive the remodeling phase.

Previous retrograde labeling studies indicate that some retinal axons make topographic targeting errors (O'Leary et al., 1986; Yhip and Kirby, 1990), but none have suggested

\footnotetext{
Received June 10, 1991; revised Oct. 28, 1991; accepted Oct. 31, 1991.

This work was supported by NEI Grant EY07025. Partial support for D.K.S. was provided by NEI Training Grant EY07057.

Correspondence should be addressed to Dr. Dennis D. M. O'Leary, MNL-O, The Salk Institute, P.O. Box 85800, San Diego, CA 92186.

Copyright (C) 1992 Society for Neuroscience $0270-6474 / 92 / 121212-21 \$ 05.00 / 0$
}

the degree of diffuseness revealed by anterograde labeling with Dil. Our findings show that directed axon growth is inadequate as a mechanism to develop the topographic ordering of retinal axons in the rat SC. Rather, mechanisms that control the removal of mistargeted axons and promote the arborization of correctly positioned axons are critical for the development of retinotopic order. These data characterizing the time course of the development of topographic order in the rat retinocollicular projection provide a framework for interpreting the potential roles that putative position-encoding molecular cues in the retina and SC might play in this process.

The retinotectal projection of nonmammalian vertebrates has been the preeminent model system for investigations of the mechanisms that contribute to the formation of precisely ordered axonal connections. Most studies have focused on the reestahlishment of a retinntopically ordered map by regenerating retinal axons in the optic tectum of frogs and fish. During regeneration, retinotopic order emerges from an initially diffuse projection (Fujisawa et al., 1982; Stuermer, 1988a). Over the past several years, emphasis has shifted to characterizing the developing retinotectal projection in frogs (Holt and Harris, 1983; Holt, 1984; Sakaguchi and Murphey, 1985) and fish (Stuermer, 1988b). These studies conclude that topographic order is present even at early stages of development and is established by the directed growth of retinal axons to their topographically appropriate regions of the tectum. A significant increase in the precision of the visual map does slowly occur, but this progressive change results from a disparate growth of the tectum relative to the size of retinal arbors, rather than by the retraction of initially large arbors or by the correction or elimination of mistargeted axons (Sakaguchi and Murphey, 1985; Stuermer, 1988h).

Studies of developing mammalian retinal projections have been hindered by limitations of the techniques available for axon labeling, but nonetheless they suggest differences between mammals and other vertebrates in the development of retinotopic order. Anterograde HRP labeling from the optic tract of mice reveals that developing retinal axons transiently have multiple branches along their length in the superior colliculus (SC), the homolog of the optic tectum (Sachs et al., 1986); although the correct termination site of the labeled axons is unknown, by inference, not all of their branches can be topographically correct. Retrograde labeling in neonatal rats shows that some ganglion cells in peripheral temporal retina send their axons caudally past the topographically correct rostral SC as far as its caudal border (O'Lcary et al., 1986; Yhip and Kirby, 1990). These studies indicate that topographic inaccuracies occur in 
the developing retinocollicular projection, but they provide only limited insight into the targeting behaviors of developing retinal axons, the mechanics of topographic map formation-axonal extension, branching, and arborization - and the subsequent remodeling of early retinal projections. These issues can be better addressed with focal retinal injections of the fluorescent carbocyanine dye 1,1'-dioctodecyl-3,3,3',3'-tetramethylindocarbocyanine perchlorate (DiI; Honig and Hume, 1989), which allow a direct labeling of developing axons originating from a defined site in the mammalian retina. In a preliminary study using this technique in perinatal rats, we found that temporal and nasal retinal axons initially project more diffusely to their primary target, the contralateral $\mathrm{SC}$, than would be predicted from the previous studies (Simon and O'Leary, 1990).

Herc, using antcrograde DiI labeling, we describe in rats the topographic targeting of retinocollicular axons arising from the periphery of each of the four retinal quadrants and from more central retina, characterize the emergence of topographic order, and present qualitative and quantitative data to provide an accurate depiction of the events and time course of this process. These data provide new insights into the mechanisms involved in the development of topographically ordered retinal projections. In addition, such information is of considerable importance for the search for antigens that may be relevant to this process. The rodent retinocollicular projection has become a prominent entity in this field; a number of molecules have been identified to be distributed in a graded or restricted fashion in the developing retina and SC, suggesting that they may have a role in the development of retinotopic maps (e.g., ConstantinePaton et al., 1986; Mendez-Otero et al., 1988; Schlosshauser et al., 1988; Rabacchi et al., 1990). A thorough characterization of the development of topographic order in retinal projections to the $\mathrm{SC}$ is essential for a meaningful interpretation of the significance of any putative position-encoding molecules.

\section{Materials and Methods}

\section{Animals}

Sprague-Dawley and Long-Evans rats ranging in age from embryonic day (E) 20 to postnatal day (P) 19, obtained from timed pregnant females (Harlan Sprague-Dawley Inc. or Sasco Inc.), were used for these experiments. The first $24 \mathrm{hr}$ following insemination is designated E0. Rats are normally born early on F.22. The first $24 \mathrm{hr}$ after hirth is designated PO.

\section{Axonal labeling methods}

A 5-10\% solution of the fluorescent lipophilic dye DiI (1,1'-dioctodecyl3, 3, 3', 3'-tetramethylindocarbocyanine perchlorate; Molecular Probes) in dimethylformamide (Sigma) was pressure injected through a glass micropipette (tip internal diameter of about $50 \mu \mathrm{m}$ ) using a Picospritzer II (General Valve Corp.). The rats were anesthetized (perinatal rats by hypothermia; older rats with open ether) and the pipette was inserted into the retina through a small hole made in the sclera with a tungsten needle. Approximately $0.01 \mu \mathrm{l}$ of DiI solution was injected. After a survival period ranging from $12 \mathrm{hr}$ to $6 \mathrm{~d}$, the rats were deeply anesthetized by hypothermia or with $3.5 \%$ chloral hydrate in $0.9 \%$ saline $(3.5 \mathrm{mg} / 10 \mathrm{gm}$ body weight) and perfused transcardially with buffered 4\% paraformaldehyde. Rats studied at embryonic ages were delivered surgically on E20 or E21, injected with DiI, and placed in a humidified incubator set at $37^{\circ} \mathrm{C}$ for $12-24 \mathrm{hr}$. They were then anesthetized and perfused as described above. All ages given in the text and in Table 1 refer to the ages at which the animals were perfused.

\section{Analysis}

Terminology. Terminology for topographic axes in the retina and the superior colliculus of mammals, or the nonmammalian homolog, the

\begin{tabular}{|c|c|c|c|c|}
\hline & \multicolumn{2}{|c|}{ Sprague-Dawley } & \multicolumn{2}{|c|}{ Long-Evans } \\
\hline & Age & Number & Age & Number \\
\hline \multirow[t]{8}{*}{ Temporal retina } & E20-E21 & 6 & $\mathrm{P} 1-\mathrm{P} 2$ & 7 \\
\hline & $\mathrm{P} 0$ & 6 & & \\
\hline & $\mathrm{P} 1-\mathrm{P} 2$ & 25 & & \\
\hline & P4 & 9 & & \\
\hline & P6 & 12 & & \\
\hline & $\mathrm{P} 8$ & 9 & & \\
\hline & $\mathrm{P} 11-\mathrm{P} 12$ & 16 & & \\
\hline & P19 & 4 & & \\
\hline \multirow[t]{5}{*}{ Nasal retina } & $\mathbf{P} 1-\mathbf{P} 2$ & 11 & $\mathbf{P} 1-\mathrm{P} 2$ & 4 \\
\hline & $\mathrm{P} 4$ & 7 & & \\
\hline & P8 & 5 & & \\
\hline & $\mathrm{P} 11-\mathrm{P} 12$ & 11 & & \\
\hline & P19 & 3 & & \\
\hline \multirow{2}{*}{ Superior retina } & $\mathrm{P} 1-\mathrm{P} 2$ & 12 & $\mathrm{P} 1-\mathrm{P} 2$ & 3 \\
\hline & $\mathrm{P} 11-\mathrm{P} 12$ & 7 & & \\
\hline \multirow[t]{2}{*}{ Inferior retina } & P1-P2 & 12 & $\mathrm{P} 1-\mathrm{P} 2$ & 1 \\
\hline & $\mathrm{P} 11-\mathrm{P} 12$ & 7 & & \\
\hline \multirow{2}{*}{$\begin{array}{l}\text { Central-temporal } \\
\text { retina }\end{array}$} & P1-P2 & 6 & $\mathrm{P} 1-\mathrm{P} 2$ & 2 \\
\hline & $\mathrm{P} 11-\mathrm{P} 12$ & 8 & & \\
\hline
\end{tabular}

optic tectum, can differ. We list the terms used in this article, and common synonyms. For retina, temporal $=$ posterior, nasal $=$ anterior, superior $=$ dorsal, inferior $=$ ventral; for $\mathrm{SC}$ (or optic tectum), rostral $=$ anterior, caudal $=$ posterior, lateral $=$ ventral, medial $=$ dorsal.

Histology. The injected retina and the dorsal midbrain, including the superior colliculus contralateral to the injected eye, were whole-mounted on glass slides and examined using rhodamine optics on an epifluorescence microscope equipped with a $100 \mathrm{~W}$ mercury lamp. Prior to whole mounting, cuts were made in the retina to maintain orientation. The sizes of the colliculi were determined directly from whole-mounts by measuring the distance along the rostral-caudal axis from the rostral border of the SC to the most distant point along its caudal edge, and the distance along the medial-lateral axis from the medial edge of the $\mathrm{SC}$ to the most distant point along its lateral edge. The size of the retina and injection site were determined from tracings of dark-field and fluorescence photographs of whole-mounts of the retinas. Some colliculi at each age were later embedded in $3.5 \%$ agar, $8 \%$ sucrose and sectioned sagittally at 250 or $300 \mu \mathrm{m}$ on a vibratome. Every section was mounted serially on glass slides and examined under fluorescence. In some cases, the optic nerve was also embedded as above and cross-sectioned at 100 $\mu \mathrm{m}$ on a vibratome.

Determination of labeling efficiency. Based on measurements of cell density in the periphery of the adult rat retina (Dreher et al., 1984), the cell density in the periphery of the retina in P2 and in P12 and older rats was estimated after compensating for age-related differences in the overall number of retinal ganglion cells due to naturally occurring ganglion cell death (Crespo et al., 1985), as well as for changes in the size of the retina. Such a calculation is valid since cell death is fairly evenly distributed across the retina in rodents (Sengelaub and Finlay, 1982). Based on the size of the injection site, these data allow an estimation of the number of retinal ganglion cells in the area covered by the DiI injection. This predicted number was compared to the number of axons actually labeled to determine the "labeling efficiency." The number of labeled axons was counted in representative P2 and P12 cases from cross sections of the optic nerves. This number provides an accurate assessment of the number of labeled retinal ganglion cells since ganglion cell axons that leave the eye do not branch in the retina or optic nerve (Perry et al., 1983; Nakamura and O'Leary, 1989; D. K. Simon and D. D. M. O'Leary, unpublished observations).

Determination of "SC coverage." In representative, well-labeled cases with a peripheral temporal DiI injection, the percentage of the $\mathrm{SC}$ area covered by a high density of labeling, termed the "SC coverage," was measured from photographs of whole mounts. This region of highdensity labeling was clearly defined at P8 and later, and corresponded 

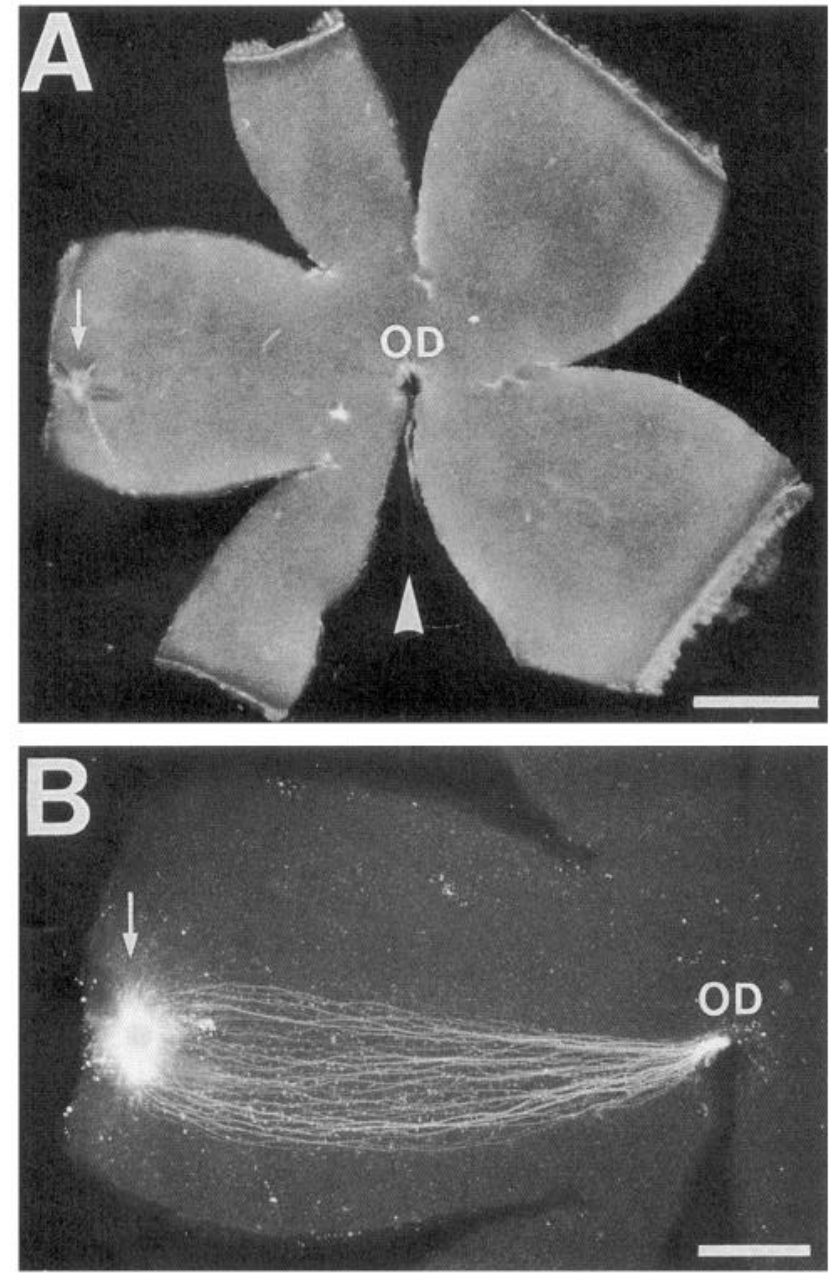

Figure 1. A representative retina and DiI injection site: low-power dark-field $(A)$ and higher-power fluorescence $(B)$ photomicrographs of a retinal whole-mount from a P2 rat injected with DiI on P0, viewed with a rhodamine filter set. The arrows indicate the injection site in peripheral nasal retina near the dorsal-ventral midline. This retina is taken from the case illustrated in Figure $5 A$. The arrowhead in $A$ marks the incision in ventral retina near the nasal temporal midline for orientation. All labeled axons entering the optic disk $(O D)$ could be traced back to the injection site. Scale bars: $A, 1 \mathrm{~mm} ; B, 500 \mu \mathrm{m}$.

to the topographically correct region of rostral SC. At P4 and P6, the precise boundaries of this region were not as well defined but could be estimated. We did not attempt to define the boundaries of this region in rats younger than P4. In each case for which the SC coverage was determined, the retinal injection of DiI covered between $0.25 \%$ and $1.0 \%$ of the retina. For each age group, the average injection size covered $0.4-0.5 \%$ of the retina.

Quantification of axon branching. In well-labeled cases with a peripheral nasal injection of DiI, the branches formed by individual labeled nasal axons in the rostral half of the SC were counted in wholemounts or $300 \mu \mathrm{m}$ sagittal sections at $250 \times$ using rhodamine optics on a fluorescence microscope. The number of branches in the rostral half of the SC was recorded for every axon that could be unambiguously traced through the entire rostral half of the SC. The shortest branches counted were $5 \mu \mathrm{m}$ in length. The length of each branch was recorded as being greater than or less than $15 \mu \mathrm{m}$. In some cases, data were collected from sagittal sections after first making counts from the whole mounts. These two methods yielded similar results, and data from both methods are combined. When both methods were used for an individual case, only the data obtained from the sagittal sections are included, since a greater number of axons could be traced in them. For each case in which sagittal sections were examined, the most lateral section was excluded since its extent is very limited along the rostral-caudal axis.

\section{Results}

\section{DiI injections}

To characterize the emergence of topographic order in the rat retinocollicular projection, we made focal injections of DiI into the retina of developing and "mature" rats to label at high resolution small numbers of ganglion cell axons in their primary target, the contralateral SC. Injections were made in the periphery of each of the four retinal quadrants, temporal, nasal, superior, or inferior, or in central-temporal retina (Table 1). Labeled axons were visualized after fixation in whole-mounts of sections of the midbrain. In mature rats, peripheral retina projects in a point-to-point fashion around the perimeter of the SC; therefore, peripheral injections allow us to assess the fullest range of inaccuracies in the topographic targeting, branching, and arborization of retinal axons. Temporal retina is topographically matched with rostral SC, nasal retina with caudal SC, superior retina with lateral SC, and inferior retina with medial SC (Siminoff et al., 1966). Therefore, DiI injected in temporal retina of a mature rat labels axons that terminate in rostral SC, but at varying medial-lateral positions depending on the location of the injection along the superior-inferior axis of the retina. Correspondingly, DiI injected in superior retina of a mature rat labels axons that terminate in lateral SC, but at varying rostralcaudal positions depending on the location of the injection along the temporal-nasal axis of the retina.

Identification of the retinal origin of the labeled axons allows us to predict accurately their correct terminal zone in the contralateral SC. A typical injection site is shown in Figure 1. We define the "correct terminal zone" (TZ) as the area of the SC that at maturity would receive connections from retinal ganglion cells labeled by the described DiI injection. In every case included in this study, the retinas were scanned at high power using rhodamine optics with an epifluorescence microscope to verify that all labeled axons that enter the optic disk arise from a defined, well-localized injection site. For more centrally located injections, the retrograde labeling of axons that pass through the injection site and arise from retinal ganglion cells peripheral to the injection occurs in some cases, but not in others. As we will discuss below, our findings indicate that these axons of passage are not labeled anterogradely well enough, if at all, to contribute to the labeling patterns observed in the SC. This potential concern is not an issue with peripheral injections.

In each case, the Dil injection covers less than $2 \%$, and in most cases less than $1 \%$, of the retinal area. The smallest DiI injections cover as little as $0.15 \%$ of the retina. The number of axons labeled varies among cases due to both the size of the injection and its labeling effectiveness. In 14 representative $\mathrm{P} 1-$ P2 cases, the percentage of well-labeled axons ranges from $11 \%$ to $71 \%$ of the predicted numbers of ganglion cells covered by the retinal injection site (percentages for each case: 11, 16, 20, $24,27,28,29,32,33,37,47,63,65,71)$. In 14 representative P12 cases, the percentage of axons well labeled ranges from $2 \%$ to $47 \%$ (percentages for each case: $2,4,5,6,7,7,7,8,11,14$, $23,30,39,47)$. In many if not all of these cases, the actual percentage of well-labeled axons is higher than these figures since our measurements of injection site size overestimate its effective size in the ganglion cell layer, thereby overestimating the predicted number of ganglion cells covered by it. DiI not only provides a high-resolution labeling of individual retinal axons but can also result in a detailed labeling of the majority of axons that arise from the injection site. We emphasize that our basic 
findings are the same across all cases regardless of the absolute number or estimated percentage of well-labeled axons and therefore are independent of these variables for the range of injection sizes used here.

\section{Targeting of temporal axons}

In adult rats, peripheral temporal retina is topographically matched with the most rostral part of the SC (Siminoff, 1966). We have previously shown that this mature relationship can be revealed by making a small injection of DiI into peripheral temporal retina at $\mathrm{P9}$ or $\mathrm{P} 10$, which labels, after a 2-3 d survival period, axons that terminate at the rostral SC margin in a small, highly focused TZ consisting of overlapping arbors. For the sizes of DiI injections used, typically $1 \%$ or less of the SC is covered by the TZ at P12. However, a similar peripheral temporal injection of DiI at perinatal ages labels a topographically diffuse projection pattern (Simon and O'Leary, 1990). Here, we characterize the transformation of the initially diffuse projection of temporal axons into a precise, retinotopically ordered one. This developmental sequence is illustrated in Figure 2 with a series of photomontages of dorsal views of SC whole-mounts following injections of $\mathrm{DiI}$ in the periphery of temporal retina at varying ages. Due to the thickness of the SC, particularly at the later time points examined, some labeled axons that lie deep in the $\mathrm{SC}$ might not be visible in whole-mounts. For this reason, and to illustrate certain features better, including the laminar distribution of the labeled axons, we present in Figure 3 sagittal sections of the same whole-mounts shown in Figure 2.

Retinal axons, which enter the SC at its rostral edge, first arrive in the contralateral SC on E16 (Bunt et al., 1983). We found that the first axons from peripheral retina reach the SC around E17-E18 (not illustrated). By E20-E21, in every case examined, DiI injections in the temporal periphery covering less than $2 \%$ of the retinal area label axons distributed over most of the contralateral SC. Temporal axons extend caudally well beyond their correct $\mathrm{TZ}$ located near the rostral SC border. Many of these "overshooting" axons enter the SC at a position along its medial-lateral axis incorrect for the predicted mediallateral location of their future $\mathrm{TZ}$, but others grow directly through the predicted location of their correct $\mathrm{TZ}$ and continue to grow caudally into topographically inappropriate regions. Some labeled axons reach the caudal margin of the $\mathrm{SC}$, a number of which turn back rostrally; others continue caudally into the inferior colliculus. Thus, a significant proportion of temporal axons mistarget along the medial-lateral or rostral-caudal SC axes, or both. At this early stage, temporal axons have also begun to extend branches at topographically correct and incorrect positions; some axons form branches at both correct and incorrect positions. Often, branches that originate in the vicinity of the predicted correct TZ extend well out of this region, contributing to the diffuseness of the early projection. Although at E20-E21 temporal axons project diffusely to the $\mathrm{SC}$, there is an increased density of labeling in a region of rostral $\mathrm{SC}$ that would include the predicted TZ but covers a much greater area of the SC than that covered by the mature $T Z$ labeled by a similar injection in a P12 rat. This increased labeling could result from a preferential, local branching of axons that pass through this region or from a bias in the targeting of primary axons to it.

Similar to E20-E2 1, at both P0 and P1-P2, temporal axons are diffusely distributed over most of, if not the entire, SC. More labeled axons reach the caudal end of the SC, and a number extend caudally well into the inferior colliculus (IC). Of those that turn at the caudal SC border and grow back rostrally, some extend as far as the rostral SC border. The rostral bias in the density of labeling is more prominent at $\mathrm{P} 2$ than at earlier stages. Labeled axons, branches, and nascent arbors are present throughout the SC, but the number gradually decreases farther from the topographically correct rostral TZ.

At $\mathrm{P} 4$, the density of branching within the predicted $\mathrm{TZ}$ and the region surrounding it continues to increase. Labeled collaterals radiate from this rostral region of increased labeling density and frequently extend for $500 \mu \mathrm{m}$, and some for considerably greater distances. Although many mistargeted axons remain, fewer labeled temporal axons are found in caudal SC. By P6, the density of labeling in the region including the correct $\mathrm{TZ}$ has increased greatly, concurrent with a decrease in the size of this region, while the density of labeling outside of this region has diminished considerably. Labeled collaterals still radiate from the region of the $\mathrm{TZ}$ but are fewer in number, and most extend for less than $200 \mu \mathrm{m}$ beyond it. Labeled temporal axons also extend well into caudal SC, but their numbers are greatly reduced compared to earlier stages. Aberrantly positioned axons present at P6 arborize in topographically inappropriate locations distant from the correct $\mathrm{TZ}$; occasionally, labeled arbors are even found near the caudal SC border.

By P8, the projection pattern begins to resemble the mature one. The region of dense labeling at the rostral SC border is restricted to a size that approaches that of the mature TZ. Few labeled temporal axons extend beyond the $\mathrm{TZ}$, and only an occasional axon enters caudal SC. Branches formed by axons outside of the $\mathrm{TZ}$ are rare and usually short. This pattern of labeling is observed in P8 rats that had been injected with DiI on P5 or P6, as well as in rats injected at P2. Thus, the labeling pattern depends on the age of the animal at the time of fixation, not on its age at the time of dye injection. At P11-P12, like P8, the arborizations of labeled axons are very dense and highly focused in the TZ. Labeled axons extending beyond the TZ are even more rare than at $\mathrm{P} 8$, as are branches on these axons. Most of these overshooting axons can be followed back to the correct TZ. In 16 P12 cases, each having substantial numbers of welllabeled axons, we found only a single labeled axon that formed an arbor at a topographically incorrect location. The distribution of labeled peripheral temporal axons in the SC of rats examined at P19 (not illustrated) is indistinguishable from that seen at P12. In four cases, no labeled axons arborized outside of the correct $T Z$. Therefore, the remodeling of the initially topographically diffuse retinocollicular projection is essentially complete by P11-P12.

At perinatal ages, then, peripheral temporal axons project diffusely over the entire $\mathrm{SC}$, but a region of increased labeling is apparent rostrally. As topographic order emerges, the density of labeling in this region increases coincident with a reduction in its size. To describe quantitatively the time course of this event as one indicator of the emergence of retinotopic order, we determined at several ages the percentage of the SC covered by the region of dense labeling, which we term the "SC coverage." Prior to $\mathrm{P} 4$, the diffuseness of the projection does not permit a nonarbitrary estimate of this area. Therefore, we limited our determinations to $\mathrm{P} 4$ and later, ages at which the region of dense labeling was sufficiently distinct that it could be estimated. Only those cases in which the region of the retina labeled by $\mathrm{DiI}$ covered between $0.25 \%$ and $1.0 \%$ of the retina were included; the average size of this region for each age group is between $0.4 \%$ and $0.5 \%$ of the retina. Within this range of in- 

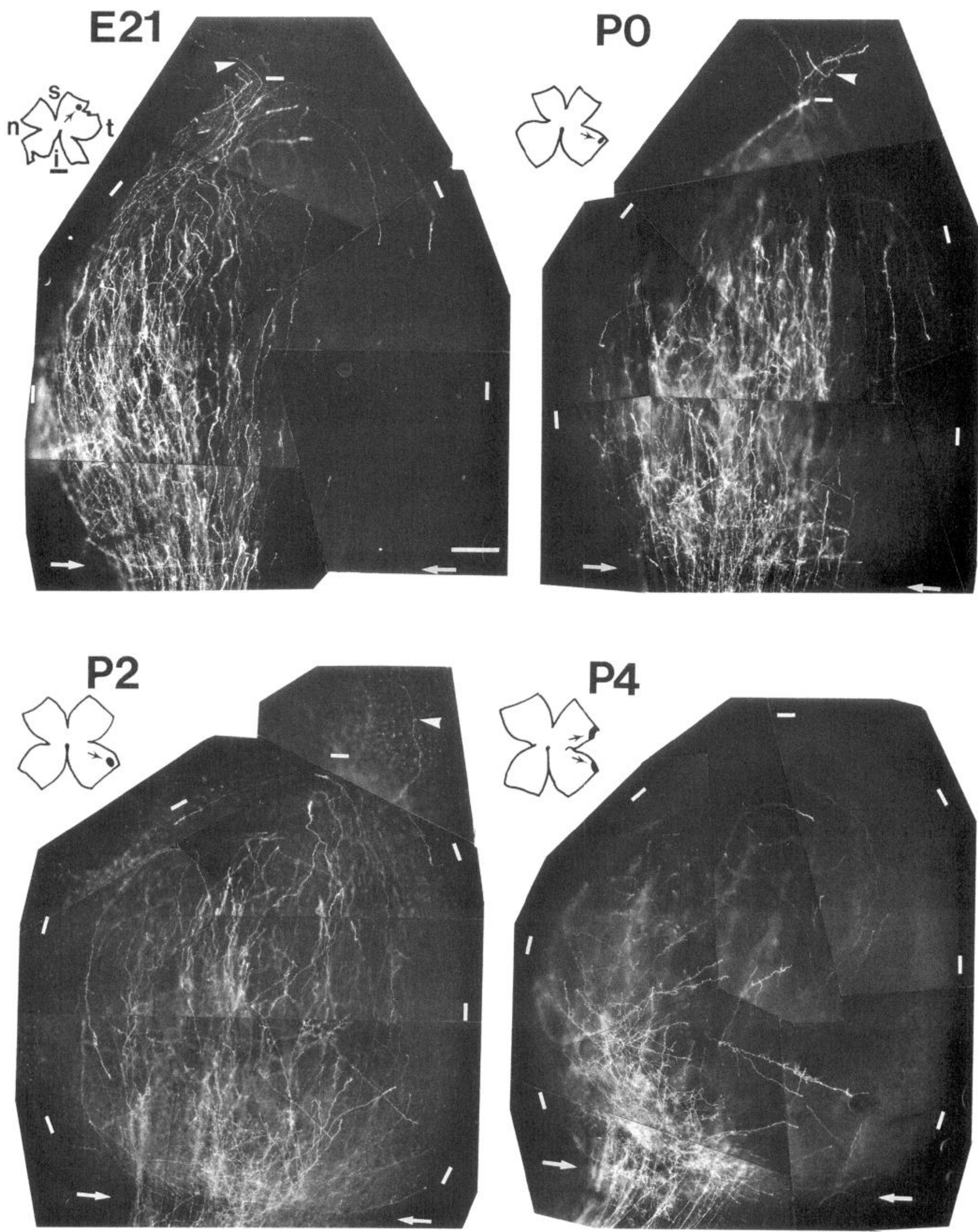

Figure 2. Gradual emergence of topographic order of temporal axons in the SC: photomontages of dorsal views of representative whole-mounts of the contralateral SC after peripheral temporal injections of DiI. The age of the animal at the time of fixation is shown beside each case. The branching and distribution in the SC of anterogradely labeled axons can be compared at each age (see Results). Dashes mark the borders of the SC. Caudal is toward the top; medial is to the right. Arrows indicate the rostral edge. Part of the IC caudal to the SC is included in those cases in 
jection sizes, the SC coverage in a given age group varies only slightly with the size of the injection. This occurs because the minimum SC coverage is limited by the size of a single axon's arbor, and because at maturity, axons arising from neighboring ganglion cells tend to form arbors that largely overlap. The SC coverage data are plotted in Figure 4. At P4 $(n=3)$, the mean SC coverage is $10 \% \pm 3.8 \%$. By P6 $(n=4)$, the SC coverage has decreased sharply to $2 \% \pm 1.1 \%$, and by P8 $(n=4)$, to $1 \%$ $\pm 0.5 \%$. At P12 $(n=5)$, the SC coverage is approximately $0.5 \%$ $\pm 0.3 \%$, the same as at P19 $(0.5 \% \pm 0.1 \% ; n=3)$, supporting our qualitative observations that the map achieves its mature topography by $\mathrm{P} 12$.

\section{Targeting of nasal axons}

Peripheral nasal axons extend across the full rostral-caudal length of the SC to reach their topographically correct $\mathrm{TZ}$ at the caudal SC margin. We assessed the targeting and branching of nasal axons at several developmental stages, 24-48 hr after making focal DiI injections in peripheral nasal retina. Representative labeling patterns from P1-P2, P4, and P12 cases are illustrated in Figure 5 with a series of SC whole mounts and in Figure 6 with sagittal sections of the same whole-mounts. A mature, topographically precise labeling pattern is present by P11-P12. At this age, the labeled axons enter the SC with a much more widespread medial-lateral distribution than that of their terminal arborizations, but the axons converge on a discrete, dense- ly labeled TZ at the caudal edge of the SC. Branches at topographically incorrect sites are rare, and no mispositioned arbors are present. This projection pattern is similar to that labeled at P19 (not illustrated), suggesting that little or no further change occurs. However, at P1-P2 nasal axons branch along their entire course through the $\mathrm{SC}$. In addition, as at maturity, many axons enter the SC at positions that are medial or lateral to the position of the correct $\mathrm{TZ}$ along this axis. As with temporal injections, though, a region of increased labeling covers a large part of the $\mathrm{SC}$ that includes, but is not restricted to, the predicted location of the relatively small correct TZ. By P4, the density of labeling within this large region in caudal SC has increased while the size of this region has decreased. Aberrant branches are still found in more rostral parts of the SC, but less frequently.

To quantify the emergence of topographic accuracy in the branching of nasal axons, we counted the number of side branches ( $\geq 5 \mu \mathrm{m}$ in length) extended by individual, labeled nasal axons in the topographically incorrect rostral half of the SC at five key ages (Fig. 7). Branches in the caudal half of the SC are more common than those in the rostral half, making it difficult to trace axons and branches in caudal SC and to quantify branch formation. It is likely that many axons that form aberrantly positioned branches in rostral SC, at the same time form both aberrantly and correctly positioned branches in caudal SC. At P2, 78\% of all labeled peripheral nasal axons have at least one branch in the rostral half of the SC and $44 \%$ have two or more

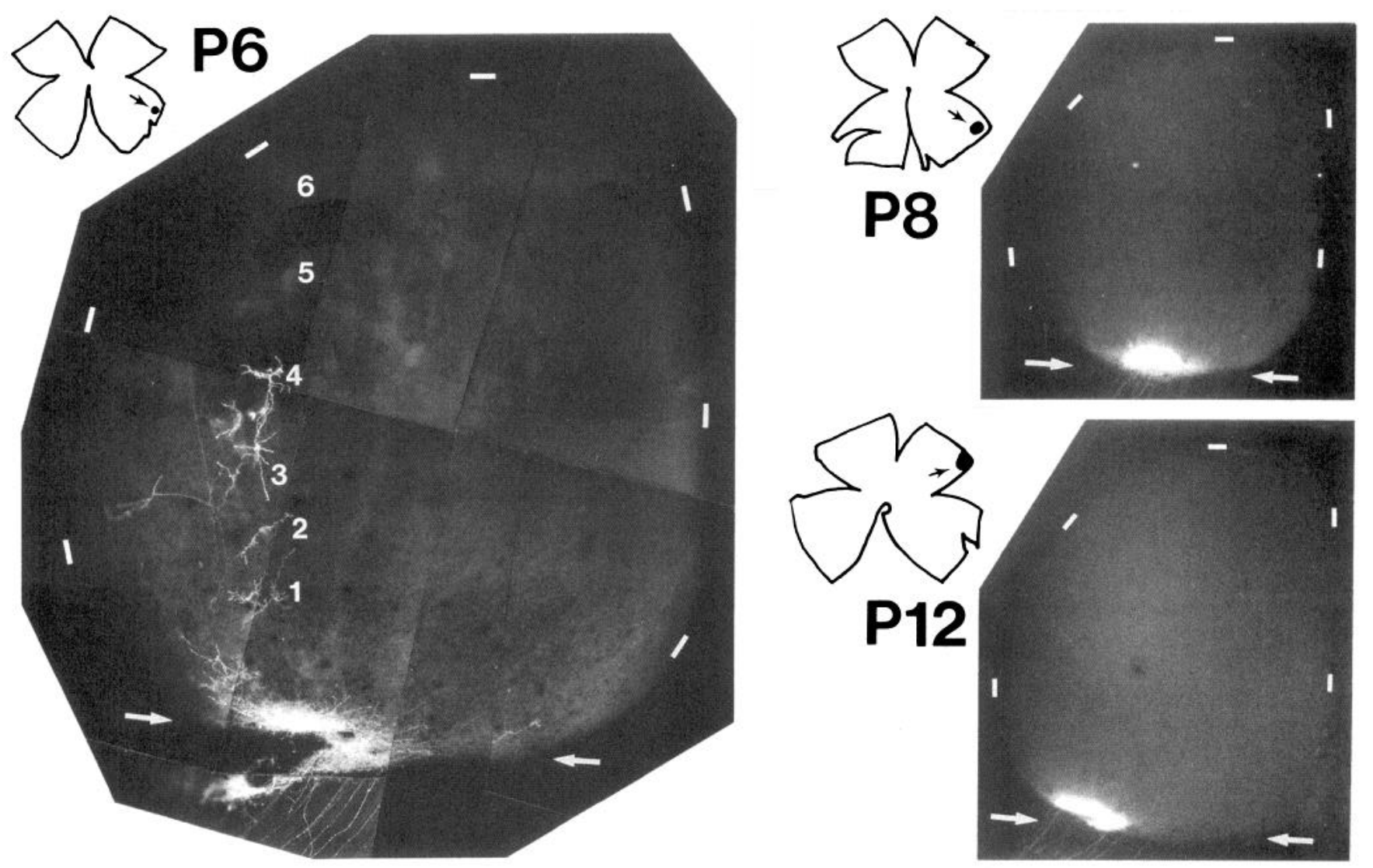

which labeled axons extended this far (E21-P2). Arrowheads indicate axons extending into the IC. An outline of the retina with the injection site indicated by an arrow is shown (in the insets) for each case. The orientation of the retina is the same for each case: $t$, temporal; $n$, nasal; $s$, superior; $i$, inferior. The small numbers over the SC of the P6 case indicate the positions of labeled arbors that are identified by corresponding numbers in the sagittal section of this case illustrated in Figure 3. The scale bar on the $E 21 \mathrm{SC}$ represents $250 \mu \mathrm{m}$ for the $E 21-P 6 \mathrm{SC}$ and $500 \mu \mathrm{m}$ for the $P 8$ and P12 SC; the retina scale bar represents $1 \mathrm{~mm}$. 

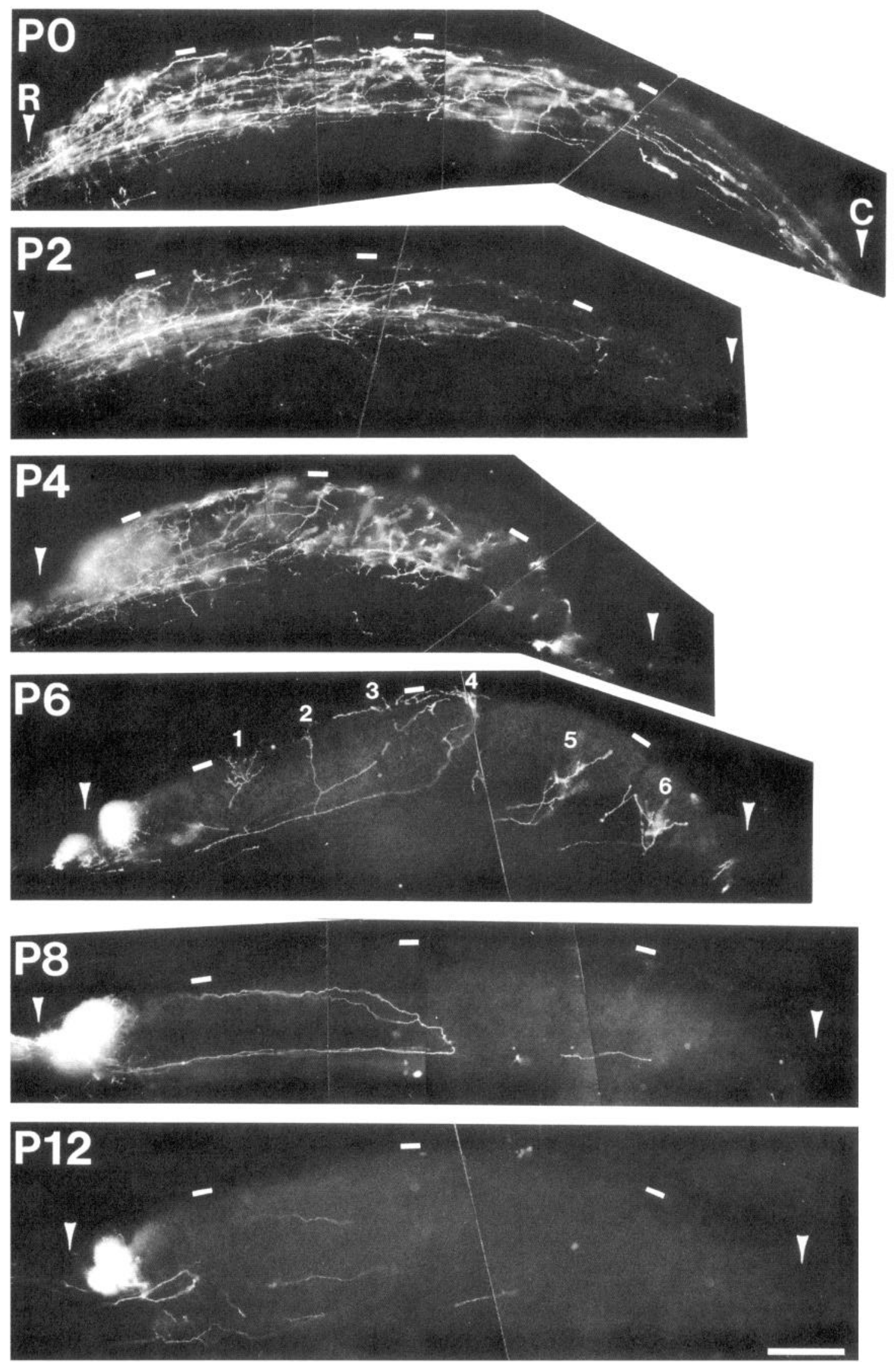

Figure 3. Targeting and branching of temporal axons in the SC: parasagittal sections $(250 \mu \mathrm{m})$ of SC after peripheral temporal injections of DiI; same cases as those illustrated in Figure 2. The rostral $(R)$ edge is indicated by the arrowhead to the left; caudal $(C)$, to the right. Dashes indicate the dorsal surface. In each case, the section illustrated was taken through the region of densest labeling in the SC; the rostral-caudal length of the 
branches (Fig. $7 A, C$ ), with an average of 1.9 rostral branches per axon. By P4, some refinement has occurred, but more than half of the nasal axons still have at least one branch in rostral $\mathrm{SC}$, with an average of 1.0 rostral branches per axon. At P8, rostral branches are found on only $24 \%$ of labeled nasal axons, with an average of 0.4 branches per axon. By P12, only $7 \%$ of nasal axons have a rostral branch, and at P19, only $4 \%$ do (this differcnce is not statistically significant). At P12 and at P19, there are only 0.1 rostral branches per axon on average. Thus, the DiI-labeled nasal axons show strong topographic specificity in the positioning of their branches at P12 and later, whereas in neonatal rats, the great majority of nasal axons form branches in the topographically incorrect half of the SC.

At the later ages examined, rostral branches on nasal axons not only are rare, but also tend to be shorter than those seen at earlier ages (Fig. 7B). 74\% of the branches present at $\mathrm{P} 2$ are greater than $15 \mu \mathrm{m}$ in length. By $\mathrm{P} 4$, half of the branches are this long, whereas only a quarter of those observed at P8 are greater than $15 \mu \mathrm{m}$ in length. At P12, only 2 of the 10 rostral branches found on 101 labeled nasal axons were greater than $15 \mu \mathrm{m}$ in length; neither of the 2 branches seen at P19 was longer than $15 \mu \mathrm{m}$. Hence, the number and mean length of rostral branches formed by nasal axons decrease concurrently. The formation, then, of a retinotopic projection of nasal axons is the net result of the elimination of topographically aberrant branches and arbors, coincident with the preferential elaboration of arbors at the correct $\mathrm{TZ}$.

\section{Targeting of superior and inferior axons}

To determine whether topographic mistargeting is common to axuns arising from all retinal regions, and to look more directly at axon targeting along the medial-lateral $\mathrm{SC}$ axis, we examined the development of the projections from peripheral superior and inferior retina. At P11-P12, axons labeled from the periphery of superior retina arborize only in a focused $\mathrm{TZ}$ at the lateralmost edge of the SC (Fig. 8, P12); similarly, axons arising from the periphery of inferior retina arborize only in a $\mathrm{TZ}$ at the medialmost edge (Fig. 9, P12). As is the case for temporal and nasal axons, axons labeled from superior or inferior retina project diffusely over much of the SC at P2 (P2, Figs. 8, 9). Although the positioning of axons is biased toward the topographically appropriate side of the SC, axons have a very widespread distribution over the medial-lateral SC axis compared to the medial-lateral width of the $\mathrm{TZ}$ that will be apparent by P12. Some axons from superior retina extend along the medial half of the $\mathrm{SC}$, and some from inferior retina extend along its lateral half. The targeting of superior and inferior axons is also diffuse along the rostral-caudal $\mathrm{SC}$ axis. In each case, many axons extend to the caudal border of the $\mathrm{SC}$, though the correct $\mathrm{TZ}$ for these axons is near the midpoint of the rostral-caudal SC axis. The branching of these axons also shows limited topographic specificity at this age. The labeled axons form branches at both topographically appropriate and inappropriate positions along the rostral-caudal and medial-lateral axes of the SC.



Figure 4. Emergence of topographic order in the arborizations of temporal axons in the SC. The percentage of the SC area occupied by a high density of labeled axons following an injection of $\mathrm{DiI}$ in temporal retina (the "SC coverage") gradually decreases postnatally. This measure is plotted for rats ages P4 $(n=3), \mathrm{P} 6(n=4), \mathrm{P} 8(n=4), \mathrm{P} 12(n=3)$, and P19 $(n=3)$. Open circles indicate individual data points; solid circles indicate the average SC coverage for each age. In each case, the percentage of the retina covered by the injection site was between $0.25 \%$ and $1.0 \%$, with an average size of $0.4-0.5 \%$ of the retina for each age group.

\section{Targeting of "central" retinal axons}

In the rat, central retinal ganglion cells tend to be generated earlier than peripheral ganglion cells and are the first to extend retinofugal axons (Morest, 1970). Therefore, axons from central retina probably reach the $\mathrm{SC}$ before those from peripheral retina and may differ from peripheral axons in their targeting bchavior. This possibility is suggested by the retrograde labeling study of Yhip and Kirby (1990), which concludes that axons arising from ganglion cells at the peripheral margin of the retina have a greater tendency to mistarget along the rostral-caudal SC axis than those from more central regions. To compare the targeting of axons from the periphery of the retina to that of axons from more central retinal regions, DiI was injected in central-temporal retina at points roughly halfway from the optic disk to the peripheral edge. At P11-P12, as expected, this results in the labeling of axons that project to a discrete, densely labeled TZ just rostral to the center of the SC (Fig. 10B,D). Few axons extend beyond this TZ, and none arborize outside of it. As with peripheral injections, though, the targeting of central-temporal axons in the $\mathrm{SC}$ at $\mathrm{P} 1-\mathrm{P} 2$ is very diffuse (Fig. 10A,C). Again,

sections varies depending on the medial-lateral position of the section. Labeled axons are diffusely distributed along the rostral-caudal axis in the younger cases. The labeling becomes progressively more restricted to a dense terminal zone at the rostral edge in the older cases. Aberrantly positioned arbors are particularly apparent at $P 6$, but not present at later stages. The small numbers over the SC of the P6 case identify labeled arbors whose positions are indicated by corresponding numbers in the whole-mount of this case illustrated in Figure 2 . Scale bar, $250 \mu \mathrm{m}$. 

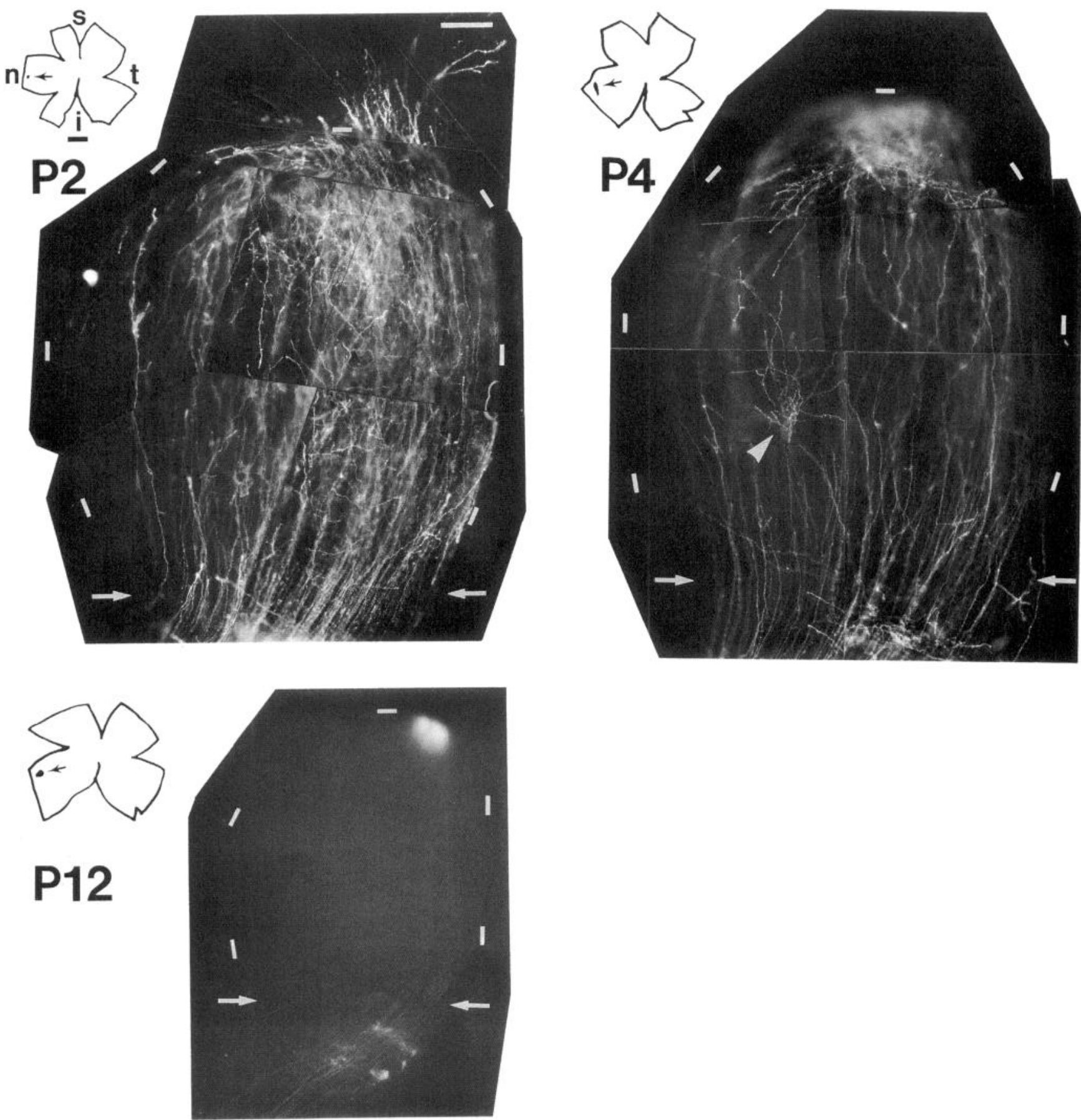

Figure 5. Gradual emergence of topographic specificity in the branching of nasal axons in the SC: photomontages of dorsal views of representative whole-mounts of the contralateral SC after peripheral nasal injections of DiI. The age of the animal at the time of fixation is shown beside each SC. Orientation is the same as in Figure 2. An outline of the retina with the injection site indicated by an arrow is shown in the insets for each case; $t$, temporal; $n$, nasal; $s$, superior; $i$, inferior. The actual injection site for the $P 2$ case is shown in Figure 1 . At $P 2$, branches of labeled axons are seen throughout the SC. An arbor is present in mid-SC in the P4 case (arrowhead). Branching at P12 is largely restricted to the terminal zone at the caudal edge. The scale bar on the P2 case represents $250 \mu \mathrm{m}$ for the P2 and P4 cases and $500 \mu \mathrm{m}$ for the P12 case; the retina scale bar represents $1 \mathrm{~mm}$.

the density of labeling is highest in a large region that surrounds and includes the predicted TZ. Many axons extend well beyond the midrostral location of the TZ, often as far as the caudal SC border, with some continuing into the inferior colliculus. Axons form numerous branches at topographically incorrect positions throughout the SC. Thus, the general targeting behavior of axons arising from more centrally located retinal ganglion cells is the same as that of axons arising from peripheral retina.

\section{Topographic targeting of retinal axons in pigmented rats}

The observations described thus far have come from SpragueDawley rats, which are albinos. Compared to pigmented rats, 



Figure 6. Targeting and branching of nasal axons in the SC: parasagittal sections $(300 \mu \mathrm{m})$ of the SC after peripheral nasal injections of DiI. The rostral $(R)$ edge is indicated by the arrowheads to the left; caudal $(C)$, to the right. Dashes indicate the dorsal surface. Labeled axons branch throughout the SC at $P 2$. Fewer rostral branches are present at $P 4$. No branches are seen in the rostral half of the $P 12$ section. Scale bar, $250 \mu \mathrm{m}$.

albino rats have a reduced ipsilateral retinocollicular projection (Lund, 1965) due to a decrease in the proportion of retinal axons that do not cross at the optic chiasm. To rule out that the topographic mistargeting of retinal axons in the $\mathrm{SC}$ is due to a defect related to albinism, we examined the targeting of axons from peripheral temporal, nasal, superior, and inferior retina, and from central-temporal retina, in neonatal Long-Evans ("hooded") rats, a pigmented strain (see Table 1). In each case, a focal injection of DiI in the retina revealed a diffuse distribution of labeled axons in the SC similar to that labeled in agematched Sprague-Dawley rats. Axons mistarget along the medial-lateral and rostral-caudal SC axes, and branch at topographically correct and incorrect sites. A representative case with a central-temporal injection is illustrated in Figure 11. The finding of a qualitatively similar diffuse retinocollicular projec- tion in neonatal pigmented and albino rats indicates that this developmental feature is not related to albinism.

\section{"Course corrections" and map remodeling}

As the initially diffuse retinocollicular projection becomes retinotopically ordered, virtually all mistargeted axons, axon segments, and branches are lost. However, evidence for earlier targeting errors is still present after the map becomes ordered. A proportion of the axons labeled from each retinal region do not take direct routes to the TZ but reach it by changing their trajectory along the rostral-caudal or medial-lateral axes of the SC. Most of the surviving axons that correct initial targeting inaccuracies are found within a few hundred microns of the TZ. Some axons correct their position by extending one or more collaterals to the TZ (Fig. 12A,B). We commonly observe axons 

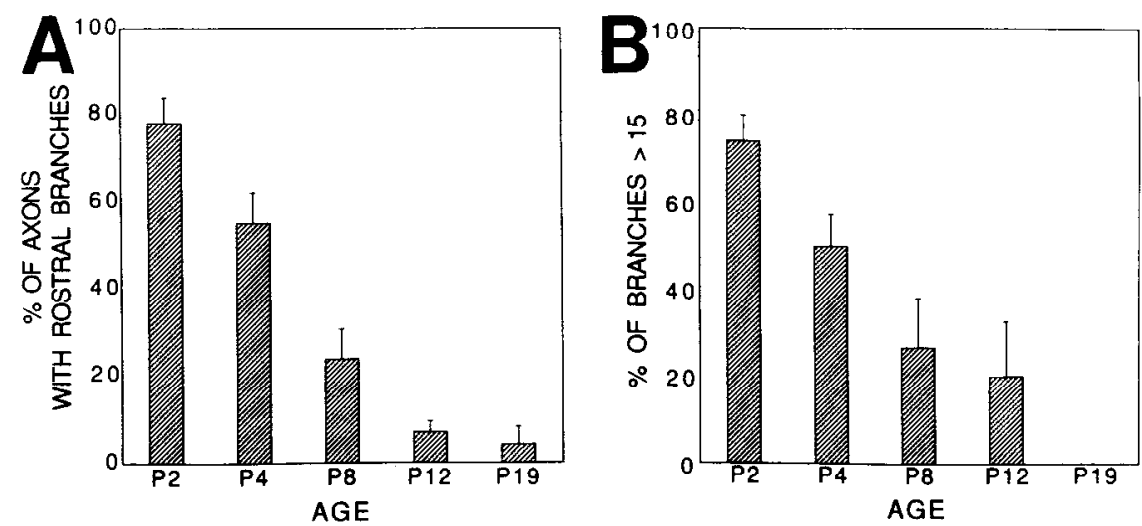

Figure 7. Quantification of changes in the aberrant branching of nasal axons in rostral SC. The number of branches (defined as an interstitial axon extension greater than or equal to $5 \mu \mathrm{m}$ in length) formed in the topographically incorrect rostral SC by individual peripheral nasal axons was determined for every labeled axon that could be unambiguously followed through the entire rostral half of the SC. Counts were made from SC whole-mounts and from $300 \mu \mathrm{m}$ sagittal sections; both methods yielded similar results. $A$, Percentage of nasal axons at various ages with one or more branches in the rostral half of the SC. $B$, Percentage of these aberrant branches that were greater than $15 \mu \mathrm{m}$ in length. $C$, The percentage of axons at $P 2, P 4$, or $P 12$ having the given number of branches in the rostral half of the $\mathrm{SC}$. The error bars indicate SE. The numbers of axons whose branches were counted were as follows: $n=50$ at $P 2$ (six rats), $n=49$ at $P 4$ (six rats), $n=$ 38 at $P 8$ (five rats), $n=101$ at $P 12$ (nine rats); $n=25$ at $P 19$ (three rats). The $P 2$ data in $B$ were obtained from 31 axons (three rats).

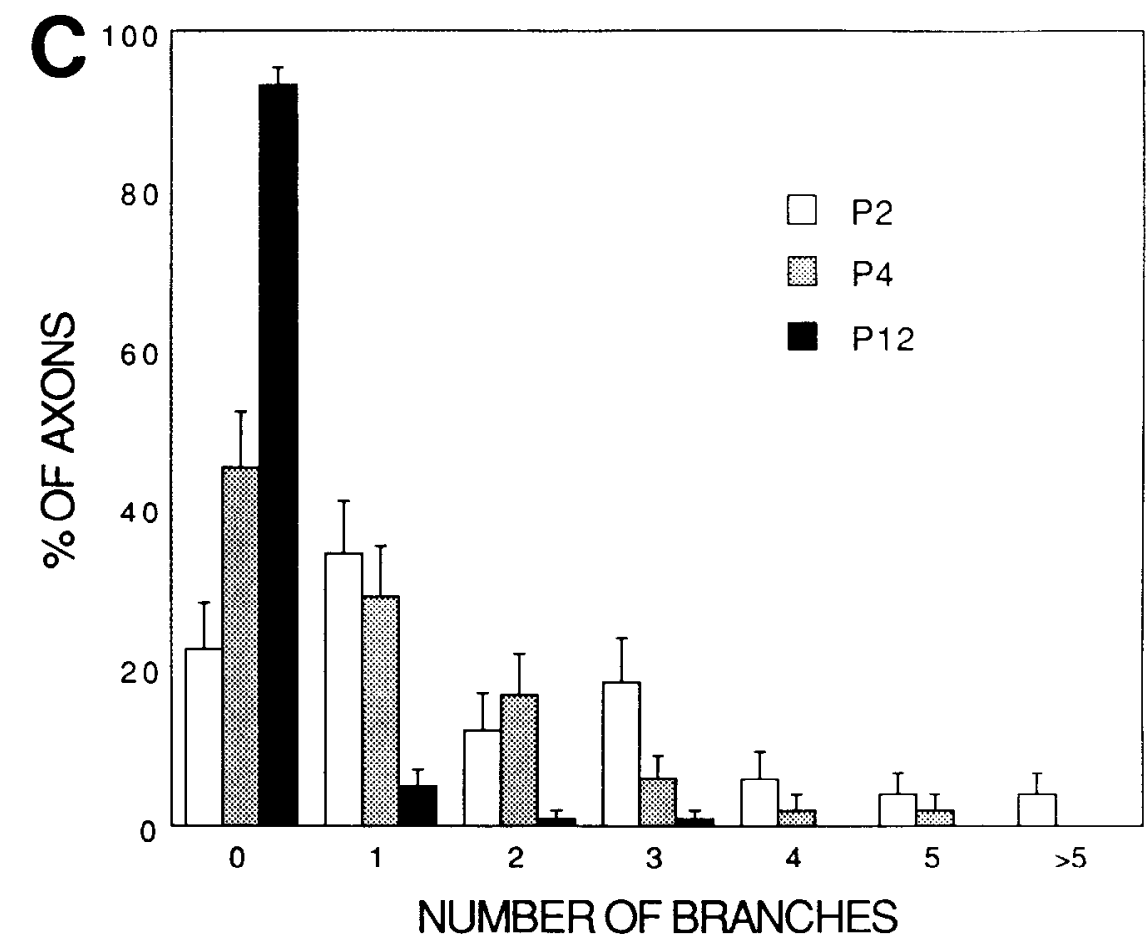

within a few hundred microns of the correct $\mathrm{TZ}$ that correct their medial-lateral position by making right-angle turns (Fig. $12 C, D)$ or by reversing their direction of growth along the rostral-caudal axis. Trajectory changes that correct much greater errors are occasionally evident. For example, in the sagittal section of the P8 case shown in Figure 3, a peripheral temporal axon extends more than a millimeter caudal to its $T Z$, makes a $180^{\circ}$ turn, and returns rostrally to end in the correct $\mathrm{TZ}$. However, long-range corrections such as this one are uncommon and not seen in every case. Because the same axon is not followed over time in this study, we cannot distinguish between a course correction accomplished by the turning of the primary axon, or by collateral extension followed by a loss of the distal segment of the primary axon. Clearly, though, mistargeted axons that pass near the TZ can correct their position through trajectory changes or collateral extension, and survive the phase of map remodeling.

\section{Relation of laminar position to axon mistargeting}

In the mature SC, retinal axons typically travel in an intermediate layer, the stratum opticum, while their arbors are restricted to a superficial layer, the superficial gray $(\mathrm{SuG})$ (Sefton and Dreher, 1985). At perinatal ages, retinal axons are dispersed in the superficialmost few hundred microns of the SC surface (Bunt et al., 1983; Sachs et al., 1986). This raises the possibility that the laminar position of retinal axons in the developing SC influences their topographic targeting. However, as is apparent in the sagittal SC sections shown in Figures 3, 6, and 10, topographically inappropriate axons and branches are found at all laminar positions. Thus, the laminar position of retinal axons in the developing SC does not appear to correlate with a propensity to topographically mistarget. The gradual displacement of the preterminal portions of retinal axons deep to the SC surface could be accounted for by the late development and expansion of the SuG (see, e.g., Altman and Bayer, 1981).

Transient extension of retinal axons to the inferior colliculus

At P2, we found that a fraction of retinal axons labeled from the periphery of each of the four retinal quadrants, and from central temporal retina, extend into the IC (see the P2 cases illustratcd in Figs. 2, 5, 8-11), confirming previous observations of a retinal projection to the IC in neonatal rodents (Bunt et al., 



Figure 8. Diffuse targeting and branching of superior retinal axons at $P 2$ compared to P12: photomontages of dorsal views of the contralateral SC after peripheral superior injections of DiI. The age of the animal at the time of fixation is shown beside each SC. At $P 2$, labeled axons extend over much of the SC, although most are located in the lateral half. A few labeled axons extended caudally from the SC into the IC. The projection is much more restricted at P12. Orientation is the same as in Figure 2. An outline of the retina with the injection site indicated by an arrow is shown in the insets for each case; $t$, temporal; $n$, nasal; $s$, superior; $i$, inferior. The DiI injection site in the $P 2$ case was split by the incision in superior retina. The scale bar on the $P 2$ case represents $250 \mu \mathrm{m}$ for the $P 2$ case and $500 \mu \mathrm{m}$ for the $P 12$ case; the retina scale bar represents 1 mm.

1983; Kato, 1983; Frost, 1984; Cooper and Cowey, 1990a,b). Some axons that reach the caudal end of the SC follow its caudal border and return rostrally, whereas others continue caudally into the IC. The labeled retinal axons rarely branch and never arborize in the IC, although the same axons that continue into the IC often branch extensively or arborize in the SC at aberrant locations. The fraction of labeled axons extending into the IC is greatest following nasal or inferior retinal injections, and smallest for superior or temporal injections. This roughly corresponds to the percentage of labeled axons from these retinal regions that reach the caudal-medial border of the SC at P2. Regardless of their retinal origin, the axons usually enter the IC medially along the SC/IC border and spread out as they extend caudally through the IC, with a tendency to curve laterally. Since the projection pattern is similar for axons labeled from each region of the retina, there is no retinotopic organization to this transient projection. Fewer retinal axons project to the IC at P4; those remaining show a similar distribution and lack of branching as at P2. By P12, labeled retinal axons are not seen in the IC, except for an occasional nasal axon that extends a short distance (less than $100 \mu \mathrm{m}$ ) into the IC. These observations suggest that the transient extension of retinal axons into the IC is a caudal continuation of retinal axons that mistarget along the rostral-caudal SC axis, rather than a novel retinal projection distinct from that which should form connections in the SC, as suggested by Cooper and Cowey (1990b).

\section{Discussion}

In adult mammals, the retina projects in a "point-to-point" fashion to the SC. Using anterograde Dil labeling, we have shown in rats that axons from the periphery of temporal, nasal, superior, and inferior retina, and from more central retinal regions, initially project diffusely in the contralateral SC, their primary target. In perinatal rats, DiI injections covering only $0.15-2 \%$ of the retina label axons distributed over much of the SC. These developing axons widely mistarget, as well as form branches and arbors at aberrant locations, along the rostralcaudal and medial-lateral axes, the two axes that define topo- 

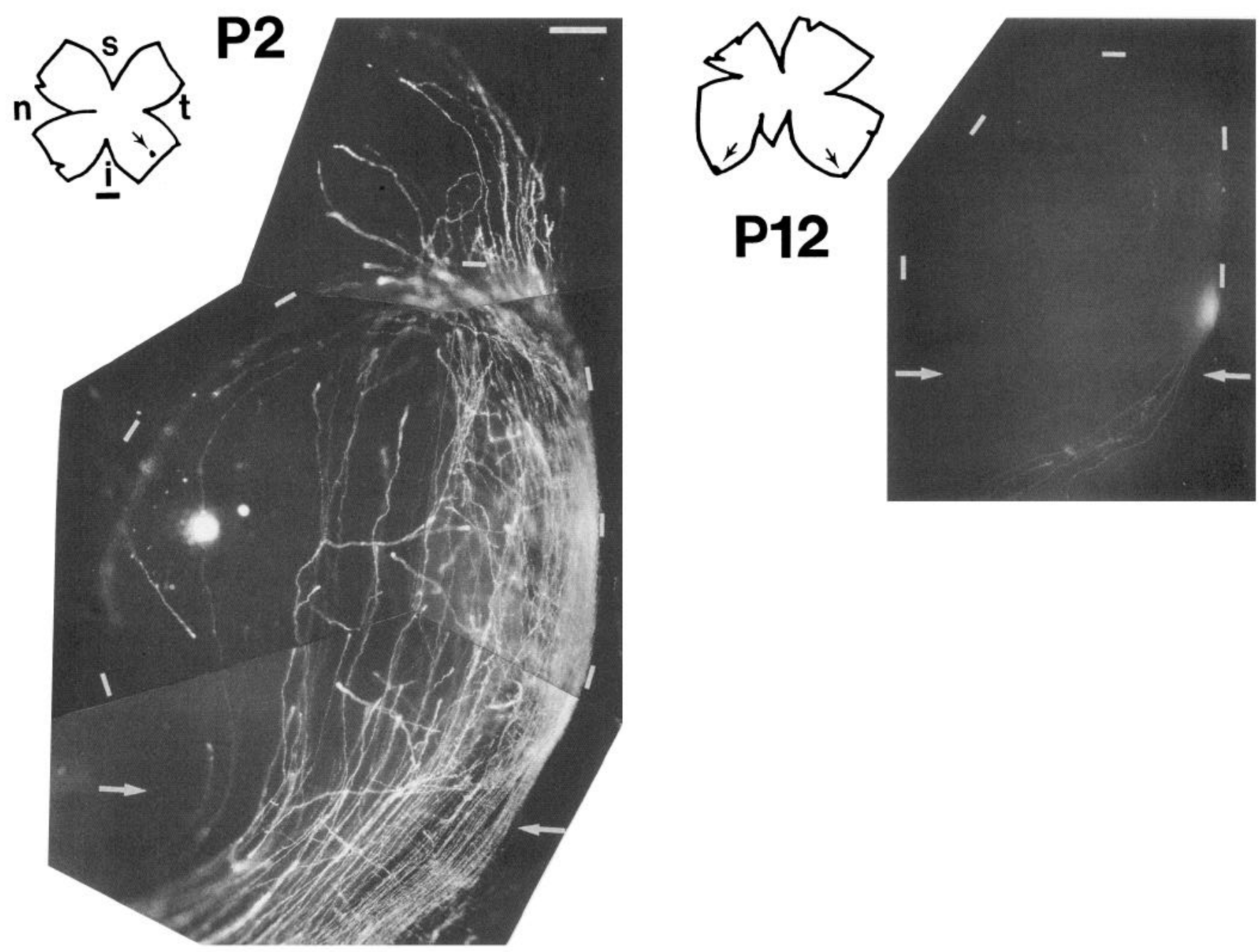

Figure 9. Diffuse targeting and branching of inferior retinal axons at $P 2$ compared to P12: photomontages of dorsal views of the contralateral SC after peripheral inferior injections of DiI. The age of the animal at the time of fixation is shown beside each SC. At $P 2$, labeled axons extend over much of the SC, but with a strong bias for the medial half. The projection is topographically ordered by P12. The IC caudal to the SC is included for the $P 2$ case. Several labeled axons extend caudally past the SC; some reach the caudal end of the IC. No axons entered the IC at $P 12$. Orientation is the same as in Figure 2. An outline of the retina with the injection site indicated by an arrow is shown in the insets for each case; $t$, temporal; $n$, nasal; $s$, superior; $i$, inferior. The DiI injection in the $P 12$ case was split by the incision in inferior retina. The scale bar on the $P 2$ case represents $250 \mu \mathrm{m}$ for the $P 2$ case and $500 \mu \mathrm{m}$ for the $P 12$ case; the retina scale bar represents $1 \mathrm{~mm}$.

graphic positions in the SC. Similar Dil injections at later stages label axons that form arborizations limited to a focused, topographically correct $\mathrm{TZ}$ covering about $1 \%$ of the SC. The directed growth of primary retinal axons toward the appropriate $\mathrm{TZ}$, if it occurs at all, is entirely inadequate as a mechanism to establish the topographic order characteristic of the mature rat retinocollicular projection. The development of topographic order is achieved by a major remodeling of the initially disordered projection involving the rapid elimination of aberrantly positioned axons, axon segments, branches, and arbors, concurrent with the preferential elaboration of arbors at topographically appropriate positions. Below, we discuss our findings, relate them to previous findings in mammals and other vertebrates, and consider their implications for molecules that may be involved in the development of topographic maps.

\section{Technical considerations}

To interpret correctly our findings on the topographic targeting of retinal axons, it is crucial to know that all labeled axons in the SC arise from a defined location in the retina or, if not, to be aware of any limitations that the axon tracing methods may impose. In principle, three technical factors could artifactually lead to the early diffuse labeling patterns that we observed: (1) direct labeling of retinal axons from ectopic labeling sites, (2) dye transfer from labeled to unlabeled axons in the optic pathway, and (3) labeling of axons passing through the injection site but arising elsewhere in the retina. We are confident that these potential factors cannot account for our results. First, ectopic DiI labeling sites were not detected in any case. Second, a number of lines of evidence indicate that dye transfer does not contribute to the labeling seen in the SC. That all labeled axons in the $\mathrm{SC}$ arise from a focal site in the retina is clearly evident in the animals fixed at P8 and later. At these ages, arborizations are limited to a densely labeled zone at a location in the SC topographically matched with the retinal injection site. Had axons from other retinal regions been labeled secondarily by dye transfer in the optic pathway, labeled arborizations would be found elsewhere in the SC, especially since, in the rat, retinal 

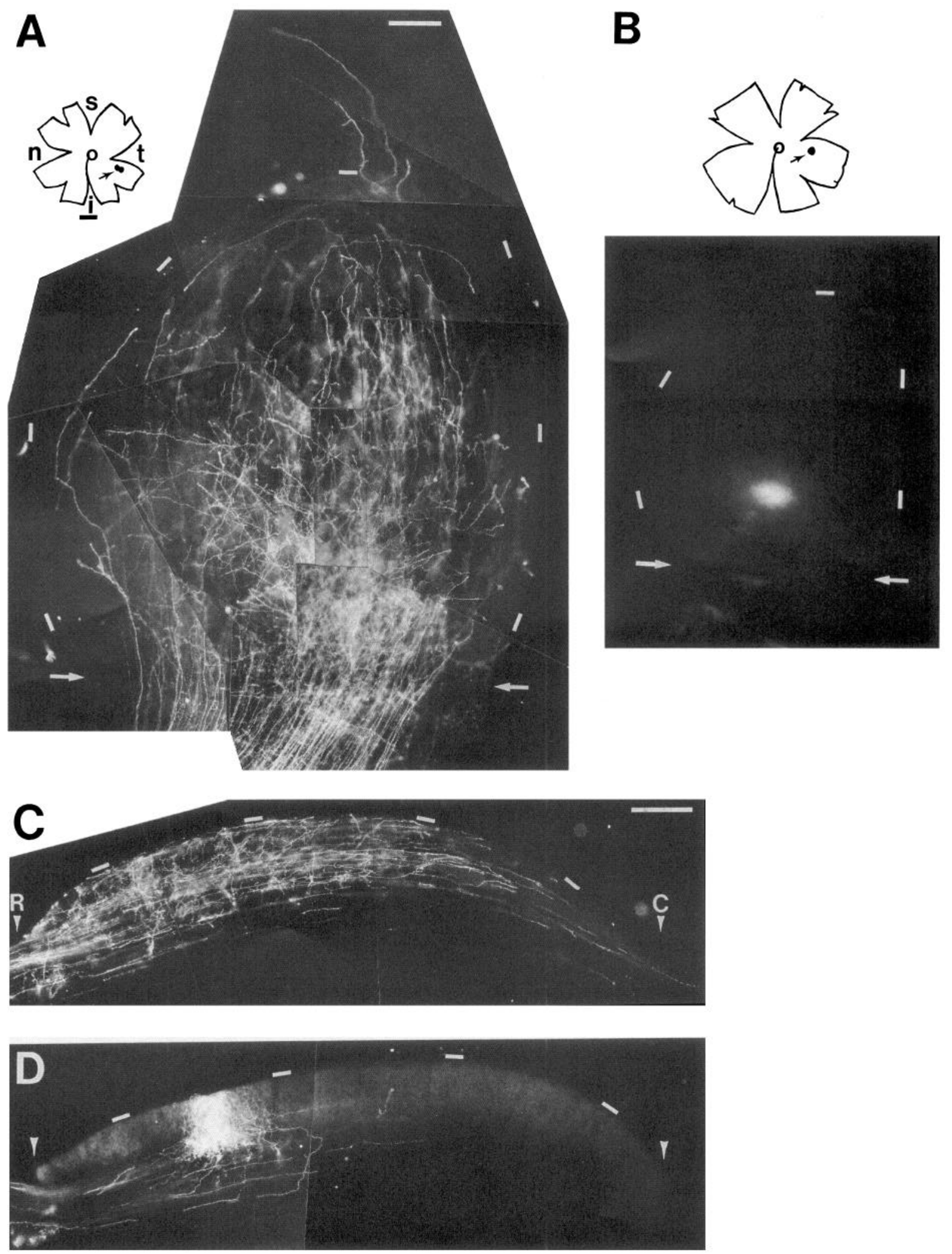

Figure 10. Targeting and branching of central-temporal retinal axons in the SC: photographs or photomontages of dorsal views $(A$ and $B)$ or sagittal sections $(C$ and $D)$ of the contralateral SC after an injection of DiI in temporal retina, midway between the optic disk (open circle) and the periphery. A, Diffuse distribution of DiI-labeled axons in the SC in a P2 rat. Many aberrantly positioned axons and branches are found throughout the SC; some axons extend caudally into the IC. B, Dense and highly focused arborizations of labeled axons in the SC of a P12 rat. At this stage, the map is topographically ordered. Very few axons remain that make targeting errors; none enter the IC. Orientation is the same as in Figure 2 . Arrowheads in $C$ and $D$ indicate the rostral $(R)$ and caudal $(C)$ borders of the SC. An outline of the retina with the injection site indicated by an arrow is shown in the insets for each case; $t$, temporal; $n$, nasal; $s$, superior; $i$, inferior. The scale bar shown in $A$ represents $250 \mu \mathrm{m}$ for $A$ and 500 $\mu \mathrm{m}$ for $B$; the scale bar in $C$ represents $250 \mu \mathrm{m}$ for $C$ and $D$; the retina scale bar represents $1 \mathrm{~mm}$. 
Figure 11. Targeting of central-temporal retinal axons in the SC of a P2 Long-Evans (pigmented) rat: diffuse distribution of DiI-labeled axons in the $\mathrm{SC}$, similar to the case illustrated in Figure $10 A$. Orientation is the same as in Figure 2. An outline of the retina with the injection site indicated by an arrow is shown in the inset; $t$, temporal; $n$, nasal; $s$, superior; $i$, inferior. An open circle indicates the position of the optic disk. SC scale bar, $250 \mu \mathrm{m}$; retina scale bar, $1 \mathrm{~mm}$.

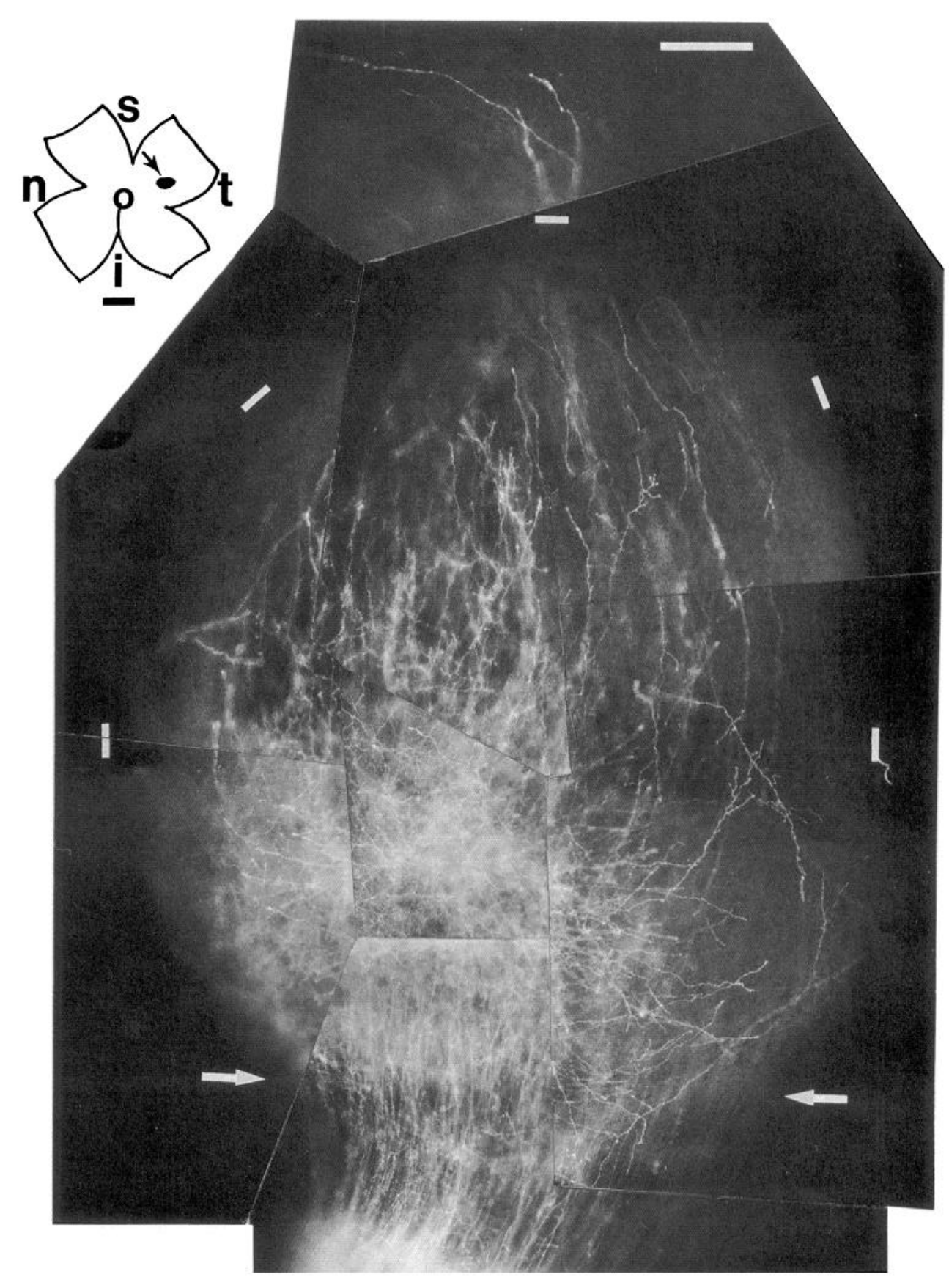

axons are not topographically ordered in the optic nerve (Simon and O'Leary, 1991). We are also confident that dye transfer between axons does not occur at younger ages. In each case, labeled axons that exit the eye travel in discrete fascicles with a similar intraretinal course from the injection site to the optic disk. Had dye transfer occurred, we would expect to see axons labeled along their intraretinal course back to parent ganglion cells scattered widely over the retina, since DiI is an excellent retrograde tracer (Honig and Hume, 1989) and retinotopic order is lost in the optic pathway (Simon and O'Leary, 1991). The most definitive evidence, though, comes from two cases injected in temporal retina at $\mathrm{P} 2$, an age when temporal axons project diffusely in the SC, but not fixed until P8. The labeling pattern in the SC of these long-term cases resembles the well-ordered projection seen in P8 cases injected at P6. Thus, the distribution of labeled axons in the SC is correlated with the age of the rat at the time of fixation, not its age at the time of DiI injection. This experiment shows that dye transfer between axons cannot explain the diffuse labeling patterns in the SC of perinatal rats. These observations are comparable to those that show that dye transfer to neighboring axons does not occur in the optic pathway of developing chicks (Nakamura and O'Leary, 1989). The third potential concern, the labeling of axons of passage, is avoided by making injections into the retinal periphery, and thus is only a potential problem with more centrally placed injections. In some of the P2 and P12 cases with central-temporal injections, a few retrogradely labeled cells are found peripheral to the injection site. However, in other cases of each 

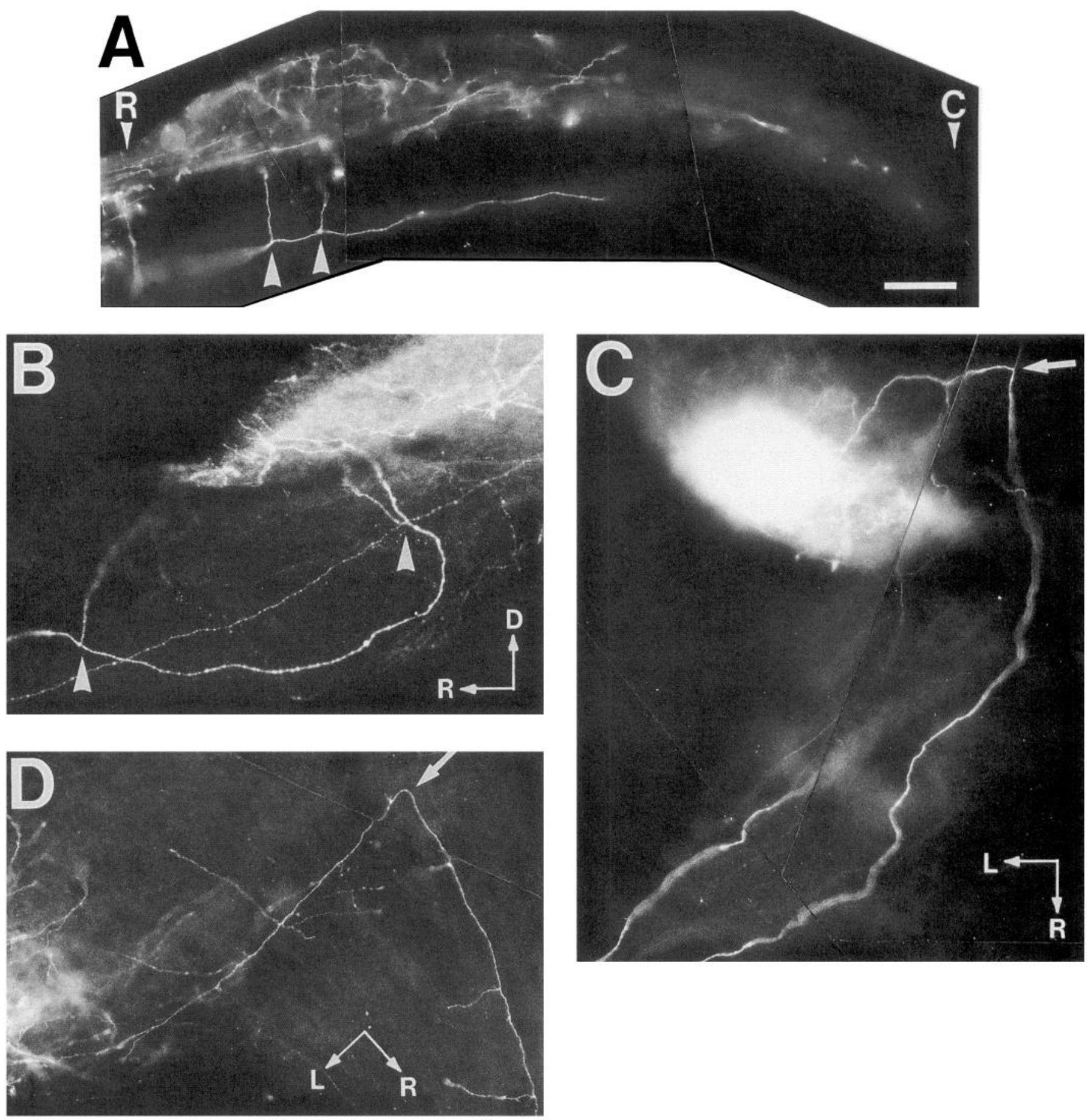

Figure 12. Targeting of retinal axons in the SC. A, Parasagittal section through lateral SC of a P2 rat following an injection of DiI in centraltemporal retina. An axon that projects aberrantly into caudal SC also extends two right-angle collaterals (arrowheads) to the superficial layers of the SC near the topographically appropriate TZ. The rostral $(R)$ and caudal $(C)$ edges of the SC are indicated. $B$, The rostral part of a sagittal section of the SC from a P6 rat following an injection of DiI in peripheral temporal retina. An axon that reaches the region of the TZ extends two branches that also reach the TZ (arrowheads). $C$ and $D$, Rostral part of a horizontal section of the SC (and optic tract) of a P11 rat (C) and a P6 rat $(D)$ following an injection of DiI in peripheral temporal-superior retina. In each case, an axon that makes a targeting error by extending into the SC medial to the TZ corrects its position by a sharp, right-angle turn (arrows). The orientation in $B-D$ is indicated for each case: $R$, rostral; $D$, dorsal; $L$, lateral. Scale bar: $A, 200 \mu \mathrm{m} ; B-D, 100 \mu \mathrm{m}$.

age group, no labeled cells are seen outside of the injection site. In both instances, though, the labeling patterns seen in the SC are the same. In the P1 2 cases, none of the labeled axons arborize outside of the well-focused, topographically correct TZ, indi- cating that axons of passsage do not contribute to the labeling in the SC. In every P2 case, the labeled axons are widely distributed over the $\mathrm{SC}$, indicating that the diffuse projection patterns observed at P2 following central-temporal injections are 
not due to the labeling of axons extended through the injection site by ganglion cells peripheral to it. When considered together, these observations show that our findings are based on the direct labeling of axons arising from a focal, well-defined, retinal injection site.

Finally, we are obligated to consider the observation that Dil does not label all of the ganglion cells estimated to be included in the injection site. Several factors probably contribute to this observation, including overestimating the size of the effective injection site, which results in an underestimate of the percentage of ganglion cell axons labeled, and mechanically damaging ganglion cells and axons with the injection pipette. Despite considerable variation in the absolute number and estimated percentage of axons labeled within each age group, we observe a diffuse retinocollicular projection in every P2 case and a topographically ordered projection in every $\mathrm{P} 12$ case. These results are consistent with previous studies using a number of different retrograde and anterograde axonal tracers to study the distribution of retinocollicular axons in developing and mature rodents, such as fast blue (O'Leary et al., 1986) and HRP (Sachs et al., 1986). The consistency of our findings indicates that they are independent of the labeling efficiency of DiI. It is possible, though, that DiI might selectively label certain classes of retinal ganglion cells. This possibility seems unlikely since carbocyanine dyes such as Dil are highly lipophilic and should partition into any cellular membrane (Honig and Hume, 1986). However, even if DiI is selective in its labeling, only a minority class(es) of ganglion cells would go undetected since we label up to $70 \%$ of the axons predicted to arise from an injection sitc in a P2 retina. In conclusion, our findings describe the behaviors representative of most, if not all, retinal ganglion cells.

\section{Development of topographic projections by retinal axons in mammals}

Retinal axons must make a number of decisions to form their correct connections. These include the choice to decussate at the optic chiasm, selection of the appropriate pathways in the brain, recognition of their target nuclei, and formation of an arbor at the topographically correct location in the target, as well as at an appropriate laminar position. In the present study, we have focused on the development of a topographically ordered projection from the retina to the contralateral SC. Previous retrograde tracing studies have indicated that developing retinal axons make topographic targeting errors in the SC (but also see Sachs et al., 1986). Injections of the retrograde tracer fast blue into caudal SC of neonatal rats labels a high density of ganglion cells in the periphery of nasal retina, and also a lower density in the temporal periphery estimated to amount to $14 \%$ of the number labeled nasally (O'Leary et al., 1986). Therefore, a significant number of ganglion cells in the temporal periphery make long-range targeting errors along the rostral-caudal SC axis since they aberrantly extend axons to caudal $\mathrm{SC}$, well beyond their correct $\mathrm{TZ}$ near the rostral SC border. Other investigators using a different retrograde tracer, rhodamine latex microspheres, labeled significantly fewer aberrantly projecting cells from the SC of neonatal rats. They concluded that ganglion cells that make topographic targeting errors represent a minor component of the neonatal rat retinocollicular projection, and that the magnitude of topographic refinement is small (Yhip and Kirby, 1990). The discrepancy between the two studies might result from the failurc of rhodaminc latex microspheres to label adequately axons that pass through the injection site. The like- lihood of this suggestion is indicated by the finding that only $11 \%$ of the total number of ganglion cells retrogradely labeled by a small injection of rhodamine latex microspheres in rostral $\mathrm{SC}$ are found outside of the small, dense focus of labeled cells in the topographically matched location in temporal retina (Yhip and Kirby, 1990). Since nasal retinal axons normally pass through rostral $\mathrm{SC}$ to reach their termination sites in caudal $\mathrm{SC}$, it can be concluded that virtually all nasal ganglion cells that send axons through the injection site are not retrogradely labeled. By analogy, then, most aberrantly projecting axons that pass through injection sites of rhodamine latex microspheres are also not labeled. In contrast, fast blue (and the closely related tracer true bluc) has been shown to result in excellent retrograde labeling of neurons that send their axons through the injection site (Sawchenko and Swanson, 1981; O'Leary and Stanfield, 1986) Therefore, fast blue injections should reveal a greater proportion of ganglion cells that make topographic targeting errors. However, even the most sensitive retrograde tracing methods provide only a limited view of the development of topographic maps. By anterogradely labeling small numbers of axons arising from a defined retinal location, we found that the topographic targeting of retinal axons is more diffuse than would be predicted from the retrograde studies. A major reason is the ability to visualize directly in the SC the distribution, branching, and arborization of axons arising from any retinal region. In addition, an analysis of smaller targeting errors is difficult with retrograde labeling. In the previous retrograde studies, O'Leary et al. (1986) did not quantify cells that made smaller axonal targeting crrors, whilc Yhip and Kirby (1990), because of the criteria used to define appropriate position in the retina, placed many aberrantly projecting cells in the category of cells with appropriately targeted axons. Our findings directly reveal the topographic diffuseness of the early, retinocollicular projection and emphasize the requirement for a large-scale remodeling of the projection to achieve the mature topographic order.

Our observations show that retinal axons arising from the periphery of each of the four retinal quadrants, temporal, nasal, superior, and inferior, pass through similar phases as they develop a topographically correct projection. Initially, axons labeled from each of these retinal sites extend and branch widely over the SC. A diffuse projection pattern is also observed for axons labeled from more central retina of perinatal rats, indicating that peripheral and central ganglion cells have similar tendencies to mistarget topographically. From these same obscrvations, we can infer that this targeting phenomenon is typical of axons that arrive to the SC over a wide time period. A proportion of the axons labeled from each retinal site extend through the entire SC and caudally into the inferior colliculus (IC). The lack of topographic order in the extension of retinal axons into the IC, and the failure of these axons to form arbors or even side branches prior to their removal argue against the suggestion of Cooper and Cowey (1990a) that this transient retinal projection plays a developmental role in bringing into register visual and auditory maps via the IC. The lack of branching of retinal axons in the IC suggests that cues that govern axon branching in the $\mathrm{SC}$ are distinct from those in the IC. Our findings suggest that the transient retinal projection to the IC simply reflects the continuation of the rostral-caudal mistargeting of retinal axons seen in the $\mathrm{SC}$.

Although the $\mathrm{SC}$ projections formed by retinal axons arising from each injection site are initially diffuse, at the carlicst times examined, an increased density of labeling is found in a region 
including, but considerably larger than, the correct $T Z$. The early widespread distributions of retinal axons suggest that many, if not all, initially fail to respond to putative, position-encoding cues hypothesized to guide axons to their correct TZ. However, the region of increased labeling density near the correct $\mathrm{TZ}$ suggests that, as a population, axons labeled from a focal retinal site do respond to putative position-encoding cues. Such concentrations of labeling could result, even in the absence of a directed growth of the primary retinal axons to the topographically correct region, either from an early bias in axon branching or from the early elimination of topographically aberrant axon segments. That the regional bias in labeling along the rostralcaudal axis is due in large part to the preferential branching of axons that pass near the $\mathrm{TZ}$ can be inferred from the labeling of axons from the nasal periphery of neonatal rats. Since all peripheral nasal axons extend through rostral $\mathrm{SC}$ to reach their correct $\mathrm{TZ}$ at the caudal SC border, much of the increased labeling in a large region of caudal SC can be attributed to preferential branching. However, we cannot rule out the possibility that a bias in the targeting of primary retinal axons along the rostral-caudal axis contributes to the increased density of labeling, especially in cases in which the labeled axons should project to more rostral SC sites. A biased distribution of labeled axons is evident along the medial-lateral SC axis and reflects their distribution in the optic tract: axons arising from superior and inferior retina are biased laterally and medially, respectively, an ordering that emerges from a distribution in the optic nerve that virtually lacks retinotopic order (Simon and O'Leary, 1991).

Over time, the branching in the region around the $\mathrm{TZ}$ increases while the frequency of topographically aberrant axons, branches, and arbors decreases. The few mistargeted axons that remain at the later stages in the remodeling of the early diffuse projection tend to branch and arborize more profusely at topographically inappropriate sites. Therefore, the elaboration of aberrantly positioned arbors appears to proceed even as most are being eliminated. The eventual loss of all topographically aberrant arbors implies that an arborization, when not in the correct $\mathrm{TZ}$, cannot be maintained, and may be incompatible with the survival of the parent ganglion cell. As the remodeling process progresses, the region of increased labeling density becomes more restricted, eventually attaining the size of the mature TZ. The virtual absence of axons making large trajectory changes to reach the mature $\mathrm{TZ}$ indicates that the primary axons that make substantial targeting errors fail to correct these errors by redirecting their growth. However, some axons that survive the phase of map remodeling do take indirect routes to the $\mathrm{TZ}$, making sharp, right-angle turns or curving gradually to correct their position along one or both of the primary axes of the SC. Axons with such trajectories are common within a few hundred microns of the TZ but are only occasionally found beyond this range. Similar trajectory changes are found in the chick, but in the chick, it is typical to find numerous axons persisting at maturity that correct positional inaccuracies of greater than 500 $\mu \mathrm{m}$ from the TZ (Nakamura and O'Leary, 1989). The relatively small course corrections made by rat retinal axons could reflect the redirected growth to the $\mathrm{TZ}$ of primary axons that initially made minor targeting errors. These trajectories could also be accounted for by the extension of collaterals from primary axons passing near the correct $\mathrm{TZ}$ but continuing caudally, followed by the elimination of the scgments of the primary axons caudal to the branch points. The feasibility of this latter scenario is indicated by our finding that rat retinal axons do extend and retain collaterals to the correct $\mathrm{TZ}$. Furthermore, in chick, many retinal axons attain their correct $\mathrm{TZ}$ in the optic tectum by a process of interstitial axon branching with the subsequent elimination of the aberrant axon segment (Nakamura and O'Leary, 1989).

The remodeling of the initially diffuse retinocollicular projection involves the preferential elimination of aberrantly projecting ganglion cells; the loss of long, topographically aberrant, segments of axons without the death of the parent ganglion cells; and the elimination of aberrant side branches and arbors formed by axons that survive into maturity. It has been estimated that more than $90 \%$ of peripheral temporal ganglion cells with aberrant projections to the caudal end of the SC die during the postnatal remodeling of the retinocollicular projection ( $O$ 'Leary et al., 1986), whereas only $35-56 \%$ of all retinal ganglion cells are estimated to die during this same period (Lam et al., 1982; Potts et al., 1982; Perry et al., 1983; Crespo et al., 1985). The small proportion of these aberrantly projecting temporal ganglion cells that survive into maturity do not maintain an axon to caudal SC (O'Leary et al., 1986). Our observations indicate a more substantial role for branch elimination without the loss of the parent axon in the development of topographic order. Although we found that axons arising from all retinal regions transiently branch at topographically aberrant locations, it was most straightforward to quantify this behavior for nasal axons. Nasal axons extend through rostral SC to reach their appropriate caudal TZ, where they ultimately form focused arbors. At neonatal ages, nasal axons form side branches along their entire length in the SC. The number of rostral branches gradually declines until it is rare to find a nasal axon with a rostral branch. The percentage of nasal axons having one or more topographically inappropriate branches in the rostral half of the SC decreases from $78 \%$ at $\mathrm{P} 2$ to $7 \%$ by $\mathrm{P} 12$ (at which age topographic order appears mature). These data actually underestimate the differences between these two ages since we did not quantify the topographically aberrant branching of nasal axons in the caudal half of the SC outside of their correct TZ. At neonatal ages, branching in this region is more extensive than in rostral SC but again is rare at P12. The decrease in the proportion of axons with aberrant rostral branches occurs concurrently with the formation of arborizations at the topographically appropriate position in caudal SC. Thus, the topographic specificity of branching increases greatly from $\mathrm{P} 2$ to $\mathrm{P} 12$.

For selective cell death alone to account for the decrease in the percentage of nasal axons with aberrant rostral branches (78\% at $\mathrm{P} 2$ vs. $7 \%$ at $\mathrm{P} 12$ ), $76 \%$ of nasal retinal ganglion cells would have to die. However, estimates of the magnitude of rat retinal ganglion cell death between $\mathrm{P} 2$ and $\mathrm{P} 12$ range from $35 \%$ to $56 \%$ (Lam et al., 1982; Potts et al., 1982; Perry et al., 1983; Crespo et al., 1985). These estimates reflect the loss of ganglion cells with projections to the SC since, in adult rats, virtually all ganglion cells project to the SC (Linden and Perry, 1983). In addition, these figures are good estimates of cell loss in peripheral retina, since, as shown in hamsters, degenerating cells are rather evenly distributed across the rodent retina, with no difference between nasal and temporal retina, and only a slightly higher rate in peripheral versus central retina (Sengelaub and Finlay, 1982). A cell death rate corresponding to the maximum estimate of $56 \%$ could, at best, decrease the percentage of nasal axons with rostral branches from $78 \%$ at $\mathrm{P} 2$ to $50 \%$ of those remaining at P12, even if the observed cell death is entirely 
limited to the aberrantly projecting population. But we find that only $7 \%$ of nasal axons have rostral branches at P12. Thus, the emergence of topographic order must depend to a large extent on the elimination of aberrantly positioned branches by axons that survive and arborize at a topographically correct site in the SC.

In this way, the development of topographic projections by retinal axons to the rat $\mathrm{SC}$ resembles the development of laminar specific arbors of retinogeniculate axons in cats. In the adult cat, retinal axons from the ipsilateral and contralateral eyes arborize in different layers of the lateral geniculate nucleus. During development, retinal axons extend side branches in multiple layers; mature arbors develop through the selective loss of side branches in inappropriate layers and the continued elaboration of branches in the appropriate layer (Sretavan and Shatz, 1984, $1986,1987)$. However, the initial projection of retinal axons to the lateral geniculate nucleus may have a greater degree of topographic organization than that to the SC (Jeffrey, 1985).

\section{Comparisons with the topographic targeting of retinal axons in} nonmammalian vertebrates

Focal DiI injections in the chick retina reveal that peripheral temporal axons initially have a widespread distribution in the tectum (Nakamura and O'Leary, 1989). As in rats, chick temporal axons enter the optic tectum at inappropriate positions along its medial-lateral axis and overshoot their correct rostral TZ along the rostral-caudal tectal axis. However, developing retinal axons project much more diffusely in the rat $\mathrm{SC}$ than in the chick tectum. This distinction is most striking along the rostral-caudal axis. In the chick, neither peripheral-temporal nor central-temporal axons extend for any significant distance into the caudal half of the tectum (Matsui and O'Leary, 1989; Nakamura and O'Leary, 1989), whereas in rats, axons arising from both sites extend to the caudal SC border. Thus, retinal axons commonly mistarget in both chicks and rats, although the relative diffuseness of early retinal projections differs between these species.

Our findings reveal both differences and similarities in the targeting of developing retinal axons in rats and in frogs or fish. During normal development, the retinotectal projection of frogs (Holt and Harris, 1983; Holt, 1984; Sakaguchi and Murphey, 1985) and fish (Stuermer, 1988b) is topographically ordered from its inception. It is reported that few or no major targeting errors like those we found in the rat occur in these species. For example, in frogs (O'Rourke and Fraser, 1986; Fujisawa, 1987) and fish (Stuermer, 1988b), temporal axons never enter the lategenerated caudal half of the tectum, which eventually is occupied by nasal axons. In contrast, in rats, developing temporal axons initially extend and branch over the entire SC. Our findings in rats show some similarities with the recent findings of O'Rourke and Fraser (1990) on the development of ordered retinal arbors in frogs. At very early stages in frog tadpoles, before caudal tectum is generated, nasal axons do transiently arborize at the caudal end of rostral tectum, resulting in a transient overlap in the positioning of nasal and temporal axons (O'Rourke and Fraser, 1990). After caudal tectum forms by an addition of newly generated neurons at the "caudal" tectal border (Gaze et al., 1974; Raymond and Easter, 1983), nasal axons shift their arbors caudally (O'Rourke and Fraser, 1990). Nasal axons that arrive after caudal tectum is generated grow directly to their topographically corrcct sitc without branching in rostral tectum (Fujisawa, 1987; Stuermer, 1988b). Therefore, the tran- sient formation of rostral branches by nasal axons in nonmammalian vertebrates may be related to the requirement of these axons to wait in rostral tectum until caudal tectum is generated. In rats, the small increase in SC size over the period during which topographic order is established occurs by "interstitial" expansion, rather than by adding new cells to its perimeter (Altman and Bayer, 1981). Nonetheless, similar mechanisms may control the removal of rostrally positioned branches on nasal axons in mammals and nonmammalian vertebrates.

Although the retinotectal projection of frogs and fish becomes topographically ordered at an early stage, there is a subsequent increase in precision of the map as the arbors of individual axons come to occupy a smaller percentage of the tectum as the animal matures. This result is not due to arbor retraction; rather, the tectum expands at a greater rate than the arbors (Sakaguchi and Murphey, 1985; Stuermer, 1988b; Stuermer and Raymond, 1989). Therefore, this process does not generate topographic order but increases topographic precision. In rats, the development of topographic order from an initially diffuse retinocollicular projection cannot be explained by expansion of the SC. Virtually all retinal ganglion cells (Morest, 1970) and their target neurons in the SC (Altman and Bayer, 1981) are generated by $\mathrm{E} 17$, and the entire retina projects to the $\mathrm{SC}$ at a time when the projection is diffuse (present results). Finally, the SC grows less than 20\% along the rostral-caudal and medial-lateral axes between the stage when the projection is topographically diffuse to when it is well ordered. Thus, the gradual increase in topographic precision in frogs and fish is a distinct phenomenon from the rapid remodeling of the initially diffuse rat retinocollicular projection.

In some ways, the development of topographic order in the rat retinocollicular projection resembles the reestablishment of order in the retinotectal projection in fish and frogs following optic nerve regeneration. In contrast to developing retinal axons in normal fish and frogs, regenerating retinal axons typically make major targeting errors in the tectum (Fujisawa, 1982; Rankin and Cook, 1986; Stuermer, 1988a). Topographic order in regenerates is established largely through the removal or correction of targeting errors and the loss of topographically aberrant arbors. Despite important differences in the establishment of retinotopic maps between normal fish and frogs and regenerates, as well as between mammals and nonmammalian vertebrates, many of the fundamental mechanisms involved in this process are likely to be conserved, though emphasized to varying degrees.

\section{Implications for the role of putative position-encoding} molecules in the development of topographic maps

Molecules that encode position are hypothesized to provide a mechanism for the directed growth of retinal axons in their central targets (Bonhoeffer and Gierer, 1984; Gierer, 1987). Most hypotheses are based on a graded or regionally specific expression of these molecules in the retina and its targets. Using monoclonal antibodies, a number of candidate antigens have been identified in the retina, and in some cases, in the chick optic tectum or rodent SC. Those found in chick include "TOP" (Trisler et al., 1981; Trisler and Collins, 1987) and "TRAP" (McLoon, 1991). In addition, two monoclonal antibodies, "Jula" and "Dolce," which are very likely directed against a configuration of the high-affinity laminin receptor (Rabacchi et al., 1990), stain retina of several species, including chick and mouse, in a regionally specific manner. In the mouse, the staining is 
restricted to peripheral superior retina at embryonic ages and is virtually gone before birth except for residual staining in the extreme peripheral edge of superior retina. Since, as our results show, the rat retinocollicular projection is still topographically diffuse as late as $\mathbf{P 4}$, it is unlikely that the molecule revealed by Jula and Dolce is directly involved in the postnatal remodeling of the early diffuse retinocollicular projection. Another antibody, though, reveals in the rat retina a dorsal-to-ventral gradient of a ganglioside, termed "JONES," that exists from E17 to at least P3 (Constantine-Paton et al., 1986), a timing consistent with a role in the development of topographic order in rat retinal projections.

In support of a role for position-encoding molecules in the development of topographic order, in vitro studies of the developing visual system of chicks, fish, and rodents demonstrate a membrane-associated activity that influences retinal axon growth in a position-related manner. Studies of neurite outgrowth from chick retinal explants on alternating stripes of membranes derived from rostral or caudal optic tectum show that a component of caudal tectal membranes is "repulsive" for temporal retinal axons (Walter et al., 1987a,b). In vivo findings that chick temporal axons initially target diffusely in rostral tectum but do not extend for any appreciable distance into the caudal half of tectum (Matsui and O'Leary, 1989; Nakamura and O'Leary, 1989) suggest that the "repulsive" caudal factor may serve to restrict temporal axons to rostral tectum. In vitro "stripe" studies of fish and mouse retina show that temporal axons in these species also prefer to grow on rostral versus caudal membrancs. In fish, the preference of temporal axons for rostral membranes persists indefinitely (Vielmetter and Stuermer, 1989), which correlates with the need to maintain topographic targeting cues since fish add retinal ganglion cells throughout life (Johns and Easter, 1977). In mice, this preference can be demonstrated for SC membranes prepared from E15 to P3 mice (Godement and Bonhoeffer, 1989). At comparable developmental ages in the rat, though, we found in vivo that temporal retinal axons commonly extend well into the caudal half of the SC. Only at later ages, as the preference of temporal axons for rostral membranes is lost in vitro (Godement and Bonhoeffer, 1989), do temporal axons become restricted to rostral SC in vivo. Presently, then, the role of the caudal, repulsive activity in normal development remains unclear, particularly in rodents. However, the demonstration of similarities across species of the in vitro responses of retinal axons to target-derived substrates despite the dramatic differences in the normal in vivo development of topographically ordered retinal projections, underscores the importance of using multiple approaches to study a range of species, and of exercising caution when making generalizations.

\section{References}

Altman J, Bayer SA (1981) Time of origin of neurons of the rat superior colliculus in relation to other components of visual and visuomotor pathways. Exp Brain Res 42:424-434.

Bonhoeffer F, Gierer A (1984) How do retinal axons find their targets on the tectum? Trends Neurosci 7:378-381.

Bunt SM, Lund RD, Land PW (1983) Prenatal development of the optic projection in albino and hooded rats. Dev Brain Res 6:149168.

Constantine-Paton M, Blum AS, Mendez-Otero R, Barnstable CJ (1986) A cell surface molecule distributed in a dorsoventral gradient in the perinatal retina. Nature $324: 459-462$.

Cooper AM, Cowey A (1990a) Development and retraction of a crossed projection to the inferior colliculus in neonatal pigmented rats. Neuroscience 35:335-344.

Cooper AM, Cowey A (1990b) Retinal topography of the neonatal crossed aberrant exuberant projection to the inferior colliculus in the pigmented rat. Neuroscience 35:345-354.

Crespo D, O'Leary DDM, Cowan WM (1985) Changes in the numbers of optic nerve fibers during late prenatal and postnatal development in the albino rat. Dev Brain Res 19:129-134.

Dreher B, Potts RA, Ni SYK, Bennett MR (1984) The development of heterogeneities in distribution and soma sizes of rat retinal ganglion cells. In: Development of visual pathways in mammals (Stone J, Dreher B, Rappaport DH, eds), pp 39-58. New York: Liss.

Frost DO (1984) Axonal growth and target selection during development: retinal projections to the ventrobasal complex and other "nonvisual" structures in neonatal Syrian hamsters. J Comp Neurol 230:576-592.

Fujisawa H (1987) Mode of growth of retinal axons within the tectum of Xenopus tadpoles, and implications in the ordered neuronal connections between the retina and the tectum. J Comp Neurol 260:127139.

Fujisawa H, Tani N, Watanabe K, Ibata $Y$ (1982) Branching of retinal axons and preferential selection of appropriate branches for specific neuronal connection in the newt. Dev Biol 90:43-57.

Gaze RM, Keating MJ, Chung SH (1974) The evolution of the retinotectal map during development in Xenopus. Proc R Soc Lond [Biol] 185:301-330.

Gierer A (1987) Directional cues for growing axons forming the retinotectal projection. Development 101:479-489.

Godement P, Bonhoeffer F (1989) Cross-species recognition of tectal cues by retinal fibers in vitro. Development 106:313-320.

Holt CE (1984) Does timing of axon outgrowth influence initial retinotectal topography in Xenopus? J Neurosci 4:1130-1152.

Holt CE, Harris WA (1983) Order in the initial retinotectal map in Xenopus: a new technique for labelling growing nerve fibers. Nature 301:150-152.

Honig MG, Hume RI (1986) Fluorescent carbocyanine dyes allow living neurons of identified origin to be studied in long-term cultures. J Cell Biol 103:171-187.

Honig MG, Hume RI (1989) DiI and DiO: versatile fluorescent dyes for neuronal labeling and pathway tracing. Trends Neurosci 12:333341.

Jeffrey G (1985) Retinotopic order appears before ocular separation in developing visual pathways. Naturc 313:575-576.

Johns PR, Easter SS (1977) Growth of the adult goldfish eye. Increase in retinal cell number. J Comp Neurol 176:331-342.

Kato $T$ (1983) Transient retinal fibers to the inferior colliculus in the newborn albino rat. Neurosci Lett 37:7-9.

I am K, Sefton AJ, Bennett MR (1982) I oss of axons from the optic nerve of the rat during early postnatal development. Dev Brain Res 3:487-491.

Linden R, Perry VII (1983) Massive retinotectal projection in rats. Brain Res 272:145-149.

Lund RD (1965) Uncrossed visual pathways of hooded and albino rats. Science 149:1506-1507.

Matsui KA, O'Leary DDM (1989) Growth and arborization of chick retinotectal axons. Soc Neurosci Abstr 15:1212.

McLoon SC (1991) A monoclonal antibody that distinguishes between temporal and nasal retinal axons. J Neurosci 11:1470-1477.

Mendez-Otero R, Schlosshauer B, Barnstable CJ, Constantine-Paton M (1988) A developmentally regulated antigen associated with neural crest and process migration. J Neurosci 8:564-579.

Morest DK (1970) The pattern of neurogenesis in the retina of the rat. Z Anat Entwicklungsgesch 131:45-67.

Nakamura H, O'Leary DDM (1989) Inaccuracies in initial growth and arborization of chick retinotectal axons followed by course corrections and axon remodeling to develop topographic order. J Neurosci 9: 3776-3795.

O'Leary DDM, Stanfield BB (1986) A transient pyramidal tract projection from the visual cortex in the hamster and its removal by selective collateral elimination. Dev Brain Res 27:87-99.

O'Leary DDM, Fawcett JW, Cowan WM (1986) Topographic targeting errors in the retinocollicular projection and their elimination by selective ganglion cell death. J Neurosci 6:3692-3705.

O'Rourke NA, Fraser SE (1986) Dynamic aspects of retinotectal map 
formation revealed by a vital-dye fiber-tracing technique. Dev Biol 114:265-276.

O'Rourke NA, Fraser SE (1990) Dynamic changes in optic fiber terminal arbors lead to retinotopic map formation: an in vivo confocal microscopic study. Neuron 5:159-171.

Perry VH, Henderson Z, Linden R (1983) Postnatal changes in retinal ganglion cell and optic axon populations in the pigmented rat. J Comp Neurol 219:356-368.

Potts R, Dreher B, Bennett MR (1982) The loss of ganglion cells in the developing retina of the rat. Dev Brain Res 3:481-486.

Rabacchi SA, Neve RL, Drager UC (1990) A positional marker for the dorsal retina is homologous to the high-affinity laminin receptor. Development 109:521-531.

Rankin ECC, Cook JE (1986) Topographic refinement of the regenerating retinotectal projection of the goldfish in standard laboratory conditions: a quantitative WGA-HRP study. Exp Brain Res 63:409420.

Raymond PA, Easter SS (1983) Postembryonic growth of the optic tectum in goldfish. J Neurosci 3:1077-1091.

Sachs GM, Jacobson M, Caviness VS Jr (1986) Postnatal changes in arborization pattern of murine retinocollicular axons. J Comp Neurol 246:395-408.

Sakaguchi DS, Murphey RK (1985) Map formation in the developing Xenopus retinotectal system: an examination of ganglion cell terminal arborizations. J Neurosci 5:3228-3245.

Sawchenko PE, Swanson LW (1981) A method for tracing biochemically defined pathways in the central nervous system using combined fluorescence retrograde transport and immunohistochemical techniques. Brain Res 210:31-51.

Schlosshauer B, Blum AS, Mendez-Otero R, Barnstable CJ, Constantine-Paton $M$ (1988) Developmental regulation of ganglioside antigens recognized by the JONES antibody. J Neurosci 8:580-592.

Sefton AJ, Dreher B (1985) The rat nervous system, Vol 1, Chap 6 , The visual system (Paxinos G, ed), pp 169-221. Sydney: Academic.

Sengelaub DR, Finlay BL (1982) Cell death in the mammalian visual system during normal development. I. Retinal ganglion cells. J Comp Neurol 204:311-317.

Siminoff R, Schwassmann HO, Kruger L (1966) An electrophysiological study of the visual projection to the superior colliculus of the rat. J Comp Neurol 127:435-444.
Simon DK, O'Leary DDM (1990) Limited topographic specificity in the targeting and branching of mammalian retinal axons. Dev Biol 137:125-134.

Simon DK, O'Leary DDM (1991) Relationship of retinotopic ordering of axons in the optic pathway to the formation of visual maps in central targets. J Comp Neurol 307:393-404.

Sretavan DW, Shatz CJ (1984) Prenatal development of individual retinogeniculate axons during the period of segregation. Nature 308 : 845-848.

Sretavan DW, Shatz CJ (1986) Prenatal development of retinal ganglion cell axons: segregation into eye-specific layers within the cat's lateral geniculate nucleus. J Neurosci 6:234-251.

Sretavan DW, Shatz CJ (1987) Axon trajectories and pattern of terminal arborization during prenatal development of the cat's retinogeniculate pathway. J Comp Neurol 255:386-400.

Stuermer CAO (1988a) Trajectories of regenerating retinal axons in the goldfish tectum. II. Exploratory branches and growth cones on axons at early regeneration stages. J Comp Neurol 267:69-91.

Stuermer CAO (1988b) Retinotopic organization of the developing retinotectal projection in the zebrafish embryo. J Neurosci 8:45134530.

Stuermer CAO, Raymond PA (1989) Developing retinotectal projection in larval goldfish. J Comp Neurol 281:630-640.

Trisler D, Collins F (1987) Corresponding spatial gradients of TOP molecules in the developing retina and optic tectum. Science 237: 1208-1209.

Trisler D, Schneider MD, Nirenberg M (1981) A topographic gradient of molecules in retina can be used to identify neuron position. Proc Natl Acad Sci USA 78:2145-2149.

Vielmetter J, Stuermer CAO (1989) Goldfish retinal axons respond to position-specific properties of tectal cell membranes in vitro. Neuron 2:1331-1339.

Walter J, Kern-Veits B, Huf J, Stolze B, Bonhoeffer F (1987a) Recognition of position-specific properties of tectal cell membranes by retinal axons in vitro. Development 101:685-696.

Walter J, Henke-Fahle S, Bonhoeffer F (1987b) Avoidance of posterior tectal membranes by temporal retinal axons. Development 101:909913.

Yhip JPA, Kirby MA (1990) Topographic organization of the retinocollicular projection in the neonatal rat. Vis Neurosci 4:313-329. 